\title{
Mathematical-Physical Properties of Musical Tone Systems
}

\author{
By \\ Bruno J. Gruber \\ (Vorgelegt in der Sitzung der math.-nat. Klasse am 23. Juni 2005 \\ durch das k. M. Peter Steinhauser)
}

\begin{abstract}
In this article the fundamental properties of the physical-mathematical laws are studied upon which music is based. Thus this article is not a study of music as such, but a study of the properties of the component elements which are the "building blocks" of music in the hands of the composers. These "building blocks" are the musical sound frequencies - the musical tones. It is the intrinsic physicalmathematical structure of the musical tones, and the interrelationships among these tones, which is studied. The physical-mathematical properties of the tones, together with the physical-mathematical laws governing the relationships between the tones, that forms the musical tone system which is discussed in this article.

The fundamental properties of musical tones (also referred to as intrinsic internal musical properties) rest upon the laws of physics and mathematics. As such, they are independent of cultural priorities regarding the perception of beauty in musical tones, even though these fundamental properties form the basis upon which the various musical systems are built. The cultural priorities find their way into music via the composers, the musicians and the public in general.

The arguments used in this article are based mostly upon physics (acoustics) and mathematics. The language used rests upon the concept of symmetries and leads to a geometrical interpretation of the basic internal properties of music in the form of a (mathematically scaled) lattice in a three-dimensional space. Each dimension represents a "rescaled octave system", based upon the three prime numbers 2, 3, and 5 . The (scaled) lattice points represent the possible musical tones - with respect to some reference tone. The various musical scales are represented by subsets of lattice points satisfying certain conditions. This geometrical picture leads to a convenient and easy interpretation of the properties of the individual musical scales, and moreover, serves
\end{abstract}


to illustrate clearly the relationships between the various musical scales. As examples, early western and modern western musical scales are treated in some detail. And, for the purpose of comparison, two eastern musical scales (Japanese Noh-scales) are discussed, in addition.

A map of the musical lattice tones from the 3-dimensional geometrical lattice space upon the line of musical frequencies leads back to the standard theory of musical tones.

A careful distinction needs to be made between the concept of a mathematical scale transformation, as it pertains to lattice points and musical ratios, and the musical scales (staff) which are used to write down musical scores (notes).

\section{Introduction}

This introduction serves as a summary of both standard and well established properties of acoustics, as well as a summary of the new results which are derived in this article based upon those properties [1].

The frequency ratios of the $\left(\frac{2}{1}\right)$-octave system can be expressed in the form

$$
\begin{aligned}
& \nu_{\operatorname{lin}}\left(n, \frac{\delta}{2 \pi} ; \nu_{0}\right)=2^{n}\left(1+\frac{\delta}{2 \pi}\right) \nu_{0}, \\
& 0 \leq \frac{\delta}{2 \pi} \leq 1, \quad n=0, \pm 1, \pm 2, \ldots \\
& \nu_{\operatorname{lin}}\left(0,0 ; \nu_{0}\right)=\nu_{0}, \\
& \nu_{\operatorname{lin}}\left(n, 1 ; \nu_{0}\right)=\nu_{\operatorname{lin}}\left(n+1,0 ; \nu_{0}\right)
\end{aligned}
$$

with $\nu_{0}$ an arbitrarily chosen sound frequency (within the range of frequencies of human hearing). Eq. (1.1) states that the range of frequencies $\nu_{\text {lin }}$ can be segmented into a set of musically equivalent intervals, called octaves, distinguished by pitch $\left(2^{n}\right)$ only. The entire set, Eq. (1.1) will be called an octave system (Fig. 1).

For a given value of $n$, the frequency interval

$$
2^{n}\left(1+\frac{\delta}{2 \pi}\right) \nu_{0}, \quad 0 \leq \frac{\delta}{2 \pi} \leq 1
$$

will be called the $n$-th octave, with $n=0$ the basic octave. The reason for this segmentation of the line $\nu$ of frequencies into (musically

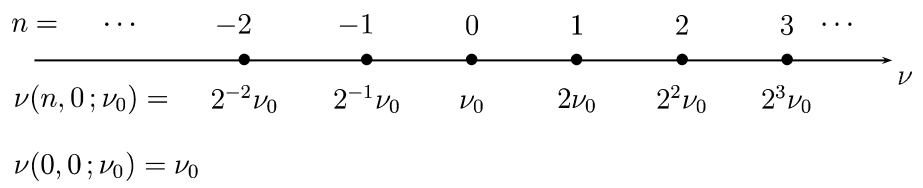

Fig. 1. The standard 2-based octave system with respect to the reference tone $\nu_{0}$ 
equivalent) octaves results from the fact that the human mind perceives the ratio of two simultaneously sounded frequencies $\nu$ and $\nu_{0}$

$$
\frac{\nu}{\nu_{0}}=2
$$

as pleasing, i.e. as a consonance.

The arbitrarily chosen frequency $\nu_{0}$ will be called the reference frequency for the octave system. The parameter $\delta / 2 \pi$ has been chosen in this form since $0 \leq \delta / 2 \pi \leq 1$ represents a cycle (Sect. 3).

An exponential form for the frequency ratios of the $\left(\frac{2}{1}\right)$-octave system is given by

$$
\nu_{\exp }\left(n, \frac{\xi}{2 \pi} ; \nu_{0}\right)=2^{n+\frac{\xi}{2 \pi}} \nu_{0}, \quad 0 \leq \frac{\xi}{2 \pi} \leq 1
$$

with

$$
\nu_{\exp }\left(0, \frac{\xi}{2 \pi} ; \nu_{0}\right)=2^{\frac{\xi}{2 \pi}} \nu_{0}, \quad 0 \leq \frac{\xi}{2 \pi} \leq 1,
$$

the basic octave. Due to the difference in the two laws for $\nu_{\text {lin }}$ and $\nu_{\text {exp }}$, the frequencies $\nu_{\text {lin }}$ and $\nu_{\text {exp }}$ differ for $\delta / 2 \pi=\xi / 2 \pi$ for all parameter values except for the values

$$
\frac{\delta}{2 \pi}=\frac{\xi}{2 \pi}=0,1
$$

for which the two frequencies agree, Fig. 2. In fact, the parameter values are related to each other by

$$
\frac{\xi}{2 \pi}=\log _{2}\left(1+\frac{\delta}{2 \pi}\right) \text {. }
$$

That is, for a given frequency $\nu_{\text {lin }}=\nu_{\text {exp }}$ the values of the two parameters $\delta / 2 \pi, \xi / 2 \pi$ disagree, or for the same parameter value $\delta / 2 \pi=\xi / 2 \pi$ two different frequencies $\nu_{\text {lin }} \neq \nu_{\text {exp }}$ are obtained, except for $\delta / 2 \pi=$ $\xi / 2 \pi=0,1$. The octave property is thus represented by

$$
\nu=\nu_{\text {lin }}\left(n, 0 ; \nu_{0}\right)=\nu_{\exp }\left(n, 0 ; \nu_{0}\right)=2^{n} \nu_{0},
$$

i.e. the frequencies for which the two frequencies agree (the consonances of the octave system based upon the reference frequency $\nu_{0}$ ).

The parameter values $\delta / 2 \pi=\xi / 2 \pi=0,1$ characterize the octave tones of the $\left(\frac{2}{1}\right)$-octave system - namely the initial points and the endpoints of each octave on the line of frequency ratios, Eq. (1.8) and 
Fig. 1. These octave tones can be looked upon as (scaled) lattice points on the line of frequency ratios.

Eq. (1.7) is a relationship of musical frequency ratios (defining musical tones) to the intervals (distances) between musical tones. That is, for two tones given by $\nu_{1} / \nu_{0}$ and $\nu_{2} / \nu_{0}$, the interval between the two tones is defined as

$$
\frac{\xi_{1}}{2 \pi}-\frac{\xi_{2}}{2 \pi}=\log _{2}\left(\frac{\nu_{1}}{\nu_{0}}\right)-\log _{2}\left(\frac{\nu_{2}}{\nu_{0}}\right)=\log _{2}\left(\frac{\nu_{1}}{\nu_{2}}\right) .
$$

Thus, the intervals for the octave tones $\nu / \nu_{0}$ of Eq. (1.8) with respect to $\nu_{0}$ are given by

$$
\frac{\xi}{2 \pi}=\log _{2}\left(\frac{\nu}{\nu_{0}}\right)=n \log _{2}\left(\frac{2}{1}\right)=n .
$$

This shows that in the lattice picture of musical tones, Fig. 1, the interval between neighboring lattice points is equal to 1 (i.e., equal to 1,200 cents), while the lattice point $n$ corresponds to the frequency ratio $\left(\frac{2}{1}\right)^{n}$.

It will be shown that, in addition to the $\left(\frac{2}{1}\right)$-octave lattice system, two "rescaled octave systems" can be introduced which are based upon the frequency ratios $\frac{3}{2}$ and $\frac{5}{3}$. These "rescaled octave systems" are given as

$$
\left(\frac{\nu}{\nu_{0}}\right)=\left(\frac{3}{2}\right)^{m} \quad \text { and } \quad \frac{\nu}{\nu_{0}}=\left(\frac{5}{3}\right)^{r} .
$$

The product of these three octave systems constitutes the musical tones which are discussed in this article, namely the frequency ratios

$$
\frac{\nu}{\nu_{0}}=\left(\frac{2}{1}\right)^{n}\left(\frac{3}{2}\right)^{m}\left(\frac{5}{3}\right)^{r} \equiv(n, m, r),
$$

$n, m, r$ integers. Eq. (1.9) can also be interpreted as representing musical lattice points in a (mathematical) 3-dimensional space.

The interval distances for the "rescaled $\left(\frac{3}{2}\right)$-octave system" are given by

$$
\frac{\xi}{2 \pi}=\log _{2}\left(\frac{3}{2}\right)^{m}=m \log _{2}\left(\frac{3}{2}\right)
$$

and for the "rescaled $\left(\frac{5}{3}\right)$-octave system" by

$$
\frac{\xi}{2 \pi}=\log _{2}\left(\frac{5}{3}\right)^{r}=r \log _{2}\left(\frac{5}{3}\right) .
$$


The familiar logarithmic formula for the intervals between musical tones of the type given by Eq. (1.9) then becomes

$$
\frac{\xi}{2 \pi}=\log _{2}\left(\frac{\nu}{\nu_{0}}\right)=n+m \log _{2}\left(\frac{3}{2}\right)+r \log _{2}\left(\frac{5}{3}\right),
$$

that is a linear expression in terms of three discrete parameters $n, m, r$. The coefficients of Eq. (1.10), $\log _{2}\left(\frac{2}{1}\right), \log _{2}\left(\frac{3}{2}\right)$ and $\log _{2}\left(\frac{5}{3}\right)$, represent the interval distances along the three lines of the $\left(\frac{2}{1}\right)$-octave, and the rescaled $\left(\frac{3}{2}\right)$ - and $\left(\frac{5}{3}\right)$-octaves. See Figs. 8, 9, 13 and 15 .

For the lattice points $\delta / 2 \pi=0$ holds and thus the corresponding frequencies are well defined and can be compared,

$$
\frac{\nu\left(n, 0 ; \nu_{0}\right)}{\nu\left(n^{\prime}, 0 ; \nu_{0}\right)}=2^{n-n^{\prime}}
$$

Since the reference tone $\nu_{0}$ can be arbitrarily chosen it is possible to associate an octave system with any musical tone, in particular also with the tones of a musical system, like the musical system of the ancient Greek Lyre, the diatonic Pythagorean musical system, etc. Considering for the moment the natural diatonic musical system, each of the tones (the German notation for the tones is used; see Sect. 6)

$\begin{array}{cccccccc}c & d & e & f & g & a & h & c^{1} \\ \nu_{0}^{\prime}=1 \nu_{0} & \left(\frac{9}{8}\right) \nu_{0} & \left(\frac{5}{4}\right) \nu_{0} & \left(\frac{4}{3}\right) \nu_{0} & \left(\frac{3}{2}\right) \nu_{0} & \left(\frac{5}{3}\right) \nu_{0} & \left(\frac{15}{8}\right) \nu_{0} & 2 \nu_{0}\end{array}$

serves as a reference tone $\nu_{0}$ for its own octave system

$$
\begin{aligned}
& \frac{\nu\left(n, 0 ; \nu_{0}=c\right)}{\left(\nu_{0}=c\right)}=2^{n}, \quad \nu\left(n, \frac{\delta}{2 \pi} ; \nu_{0}=c\right)=2^{n}\left(1+\frac{\delta}{2 \pi}\right) \cdot\left(\nu_{0}=c\right), \\
& \frac{\nu\left(n, 0 ; \nu_{0}=d\right)}{\left(\nu_{0}=d\right)}=2^{n}, \quad \nu\left(n, \frac{\delta}{2 \pi} ; \nu_{0}=d\right)=2^{n}\left(1+\frac{\delta}{2 \pi}\right) \cdot\left(\nu_{0}=d\right), \\
& \frac{\nu\left(n, 0 ; \nu_{0}=e\right)}{\left(\nu_{0}=e\right)}=2^{n}, \quad \nu\left(n, \frac{\delta}{2 \pi} ; \nu_{0}=e\right)=2^{n}\left(1+\frac{\delta}{2 \pi}\right) \cdot\left(\nu_{0}=e\right),
\end{aligned}
$$

with all the tones $2^{n} \nu_{0}$ properly defined $(\delta / 2 \pi=0)$, i.e. consonances. It holds then

$$
\frac{\nu\left(n, 0 ; \nu_{0}^{\prime}\right)}{\nu\left(n, 0 ; \nu_{0}\right)}=\frac{\nu_{0}^{\prime}}{\nu_{0}}
$$

Thus, the ratio of the $\nu_{0}^{\prime}$ octave system with respect to the $\nu_{0}$ octave system exists and is given by the ratio of the reference frequencies. 
The internal musical properties of a musical system can be expressed as ratios between octave systems. For the case of the example given by Eq. (1.12) it holds $\nu_{0}=c=1$, and $\nu^{\prime}$ is any of the 8 tones.

Since early on in time (Chinese sources $\sim 600$ B.C., Pythagoras $\sim 500$ B.C.) it has been known that the ratios of the set of numbers ([1] and [2]),

$$
\{1,2,3,4,5,6\}
$$

yield the consonances, imperfect and perfect, "within an octave".

It will be shown that the (smaller) set of the three prime numbers

$$
\{2,3,5\}
$$

forms a basis for the intrinsic musical properties of 3-parameter musical systems. It are the ratios of the 2-, 3-, 5-based octave systems from which intrinsic musical properties derive. The musical system can formally be represented by a 3-dimensional lattice $(n, m, r)$ with the lattice points corresponding to the musical tones, Fig. 15. The various musical scales form subsystems of the general system. Thus, the subsystem $(n, 0,0)$ corresponds to the standard octave system, Fig. 1. The subsystem $(n, m, 0)$ contains the ancient Greek Lyre and the Pythagorean musical system, Figs. 7 and 8. The other musical systems are contained in $(n, m, r)$ proper.

\section{Assumptions}

The following assumptions are made:

(a) The sound frequencies considered, as far as they apply to music, are always understood to be limited to the range of the frequencies of the musical instruments $\left(c^{-3}\right.$ to $c^{5}$, i.e. $2^{4} \mathrm{~Hz}$ to $\left.2^{12} \mathrm{~Hz}\right)$.

(b) The linear change of sound frequencies $\nu_{\text {lin }}$ is given by

$$
\nu_{\text {lin }}\left(\alpha, \nu_{0}\right)=\alpha \nu_{0}, \quad 0 \leq \alpha<\infty,
$$

$\nu_{0}$ some arbitrarily chosen (reference) frequency and $\alpha$ a real parameter. Then $\nu_{\text {lin }}$ represents all frequencies among which the sound frequencies are contained.

(c) The exponential change of sound frequencies is given by

$$
\nu_{\exp }\left(\beta, \nu_{0}\right)=2^{\beta} \nu_{0}, \quad 0 \leq \beta<\infty,
$$

$\beta$ a real parameter. Thus for a given value $\nu_{0}$, and for a given frequency $\nu_{\text {lin }}=\nu_{\text {exp }}$ the values of the parameters $\alpha$ and $\beta$ will be different (except for $\alpha=1, \beta=0$ and $\alpha=2, \beta=1$ ). 
(d) The human mind perceives two distinct tones $\nu$ and $\nu^{\prime}$ (sound frequencies), which are heard simultaneously, as agreeable (consonant) if they differ by a factor of 2 ,

$$
\nu / \nu^{\prime}=2
$$

for any chosen reference frequency $\nu^{\prime}$.

Choosing $\nu^{\prime}=\nu_{0}$, the frequencies $\nu$ of this interval are given by

$$
\nu \in\left[\nu_{0}, 2 \nu_{0}\right]=\nu_{0}[1,2] \quad \text { or } \quad \frac{\nu}{\nu_{0}} \in[1,2]
$$

where the square brackets denote the closed interval $1 \leq \nu / \nu_{0} \leq 2$. The interval Eq. (2.4), for arbitrarily chosen $\nu_{0}$, corresponds to the primary (basic) octave, with the two endpoints of the interval

$$
\nu=\nu_{0}, 2 \nu_{0} \quad \text { or } \quad \frac{\nu}{\nu_{0}}=1,2
$$

forming a perfect consonance.

Taking the consonance property of the octave into account, Eq. (2.1) can be rewritten in the form (Eq. (1.1)),

$$
\nu_{\text {lin }}\left(\frac{\delta}{2 \pi}, \nu_{0}\right)=\nu_{0}\left(1+\frac{\delta}{2 \pi}\right), \quad 0 \leq \frac{\delta}{2 \pi} \leq 1,
$$

where $\delta / 2 \pi$ is a real parameter. The values $0 \leq \delta / 2 \pi \leq 1$ then cover the frequencies of an octave.

Since $\nu_{0}$ can be arbitrarily chosen, the endpoint of the octave Eq. (2.6) can also be chosen as the starting point of an octave, namely the octave

$$
\nu_{\text {lin }}\left(\frac{\delta}{2 \pi}, 2 \nu_{0}\right)=2 \nu_{0}\left(1+\frac{\delta}{2 \pi}\right), \quad 0 \leq \frac{\delta}{2 \pi} \leq 1,
$$

and, since moreover the choice of $\nu_{0}$ does not affect the octave property (perfect consonance), the octave Eq. (2.7) has again the properties of the octave (2.6), except for a difference in pitch, $\nu_{0}$ being replaced by $2 \nu_{0}$ (a rescaling). The line of all sound frequencies $\nu$ can thus be segmented into octaves which have identical musical properties, except for the pitch (a rescaling of the basic interval $\nu_{0}[1,2]$ )

$$
\begin{aligned}
& \nu_{\text {lin }}\left(n, \frac{\delta}{2 \pi} ; \nu_{0}\right)=2^{n} \nu_{0}\left(1+\frac{\delta}{2 \pi}\right), \\
& n=0, \pm 1, \pm 2, \ldots, \quad 0 \leq \frac{\delta}{2 \pi} \leq 1,
\end{aligned}
$$

where $n=0$ represents the basic octave. Note that due to the octave property the negative values $n=-1,-2,-3, \ldots$ can be ignored 
since for any value $2^{-n}$ the octaves can be rescaled by the factor $2^{n+m}$

$$
2^{n+m} \cdot 2^{-n} \nu_{0}\left(1+\frac{\delta}{2 \pi}\right)=2^{m} \nu_{0}\left(1+\frac{\delta}{2 \pi}\right), \quad m=0,1,2, \ldots
$$

reproducing musically identical octaves (except for the pitch). Thus, henceforth the values for the integer $n$ will generally be restricted to nonnegative integers. It is important to note that the musical properties of all octaves are the same because they are given as ratios of frequencies. Thus the scaling factors cancel and the ratios remain the same.

For the parameters $\beta$ of Eq. (2.2) the form $\xi / 2 \pi$ will be chosen. Then the octave property applied to Eq. (2.2) yields

$$
\nu_{\exp }\left(n, \frac{\xi}{2 \pi} ; \nu_{0}\right)=\nu_{0} 2^{n+\xi / 2 \pi}, \quad 0 \leq \frac{\xi}{2 \pi} \leq 1, \quad n=0,1,2, \ldots
$$

again segmenting the frequencies into octave intervals of identical musical properties, except for the pitch.

The notation $\delta / 2 \pi$ and $\xi / 2 \pi$ for the parameters $\alpha$ and $\beta$ has been adopted in view of their significance to symmetry cycles (Sect. 3 ).

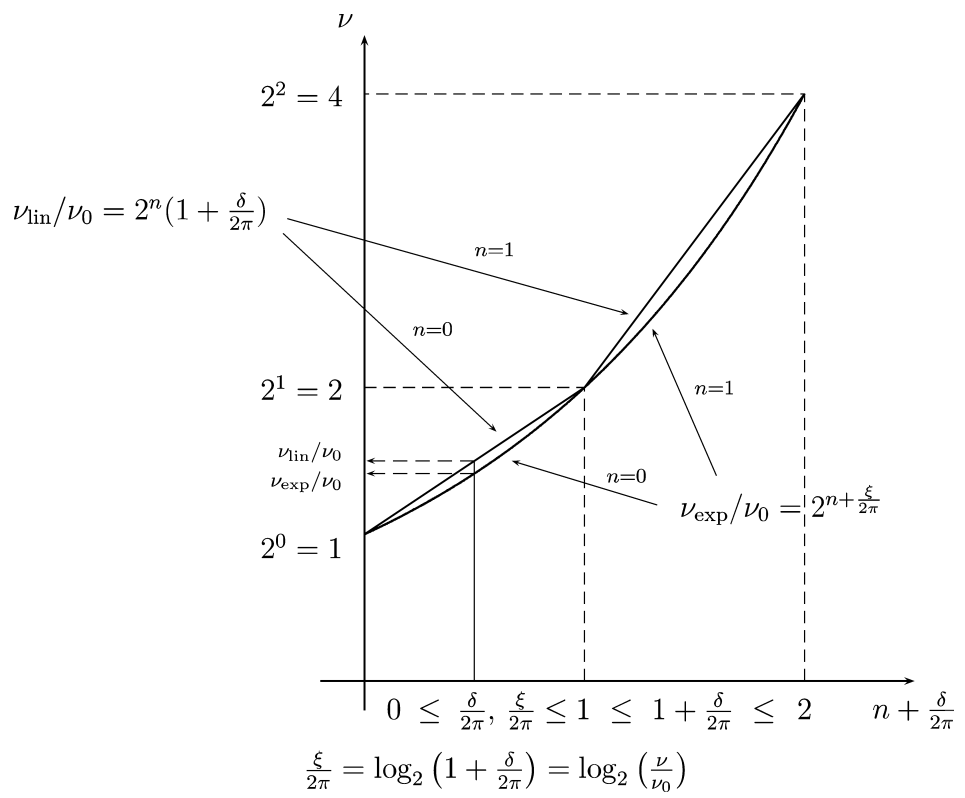

Fig. 2. The curves $\nu_{\text {lin }}$ and $\nu_{\text {exp }}$ as functions of the parameter $n+(\delta / 2 \pi)$. It holds that $\nu_{\text {lin }} / \nu_{0}=\nu_{\exp } / \nu_{0}$ if, and only if, $\delta / 2 \pi=\xi / 2 \pi=0,1$. These two values define the octave tones. For all other values $0 \leq \delta / 2 \pi, \xi / 2 \pi<1$ the two frequencies $\nu_{\text {lin }}$ and $\nu_{\text {exp }}$ differ 
(e) The two frequencies $\nu_{\text {lin }}$ and $\nu_{\text {exp }}$ given by Eq. (2.8) and Eq. (2.10) agree for the parameter values

$$
\frac{\delta}{2 \pi}=\frac{\xi}{2 \pi}=0,1
$$

These parameter values define a lattice tone. For all other parameter values there is a discrepancy between the frequency $\nu_{\text {lin }}$ and the frequency $\nu_{\text {exp }}$ (Fig. 2).

(f) The octave property (consonance of the tones $\nu$ and $2 \nu$ ) is assumed to be universally true, all humans having essentially the same receiving and analysing mechanism in their ear. The interpretation of the analysed signal in the human mind will however depend upon cultural influences. After all, the interpretation of the ratios of frequencies $\frac{5}{3}, \frac{5}{4}, \frac{4}{3}$ as consonances or as dissonances has changed with time. Thus, while cultural influences do lead to different types of music, reflecting itself in different musical scales and involving different tones, the underlying musical structure upon which the various types of music are built remains the same.

\section{Cycles and Symmetries}

If the frequencies are set to be equal it holds

$$
\begin{gathered}
\nu=\nu_{\text {lin }}=\nu_{\exp }=2^{n} \nu_{0}\left(1+\frac{\delta}{2 \pi}\right)=2^{n} \nu_{0} 2^{\frac{\xi}{2 \pi}}, \\
0 \leq \frac{\delta}{2 \pi}, \quad \frac{\xi}{2 \pi} \leq 1, \quad n=0,1,2, \ldots
\end{gathered}
$$

with

$$
\frac{\xi}{2 \pi}=\log _{2}\left(1+\frac{\delta}{2 \pi}\right), \quad 0 \leq \frac{\delta}{2 \pi}, \quad \frac{\xi}{2 \pi} \leq 1
$$

(the inverse function to Eq. (3.1)).

The octave property, that is the equivalence of the endpoints of an octave interval, combined with the equivalence of all octaves of the octave system, Eq. (2.8), suggests to look upon the octaves as cycles. The endpoint of the $n$-th octave,

$$
\nu=2^{n} \nu_{0}\left(1+\frac{\delta}{2 \pi}\right), \quad \frac{\delta}{2 \pi}=1,
$$

is also the beginning point of the $(n+1)$-st octave,

$$
\nu=2^{n+1} \nu_{0}\left(1+\frac{\delta}{2 \pi}\right), \quad \frac{\delta}{2 \pi}=0,
$$


which, in turn, is equivalent to the beginning point of the $n$-th octave (except for a scaling factor),

$$
\nu=2^{n} \nu_{0}\left(1+\frac{\delta}{2 \pi}\right), \quad \frac{\delta}{2 \pi}=0 .
$$

Thus, each octave can be represented as a frequency cycle

$$
\begin{gathered}
\exp \left[i 2 \pi \frac{\nu}{\nu_{0}}\right]=\exp \left[i 2 \pi 2^{n}\left(1+\frac{\delta}{2 \pi}\right)\right]=\exp \left[i 2 \pi 2^{n} \frac{\delta}{2 \pi}\right], \\
\exp \left[i 2 \pi 2^{n}\right]=1 .
\end{gathered}
$$

Eq. (3.3) maps the $n$-th octave upon a circle $\exp [i 2 \pi \phi], 0 \leq \phi \leq 1$, such that for each value $m$ one cycle is completed,

$$
\frac{1}{2^{n}}(m-1) \leq \frac{\delta}{2 \pi} \leq \frac{1}{2^{n}} m, \quad m=1,2,3, \ldots, 2^{n} .
$$

That is, the $n$-th octave loops the circle $2^{n}$ times (the scaling factor).

The exponent $n+(\xi / 2 \pi)$ in Eq. (3.1) describes for $0 \leq \xi / 2 \pi \leq 1$ the nonlinear (exponential) behaviour of the $n$-th octave. This exponent can thus be used to characterize the $n$-th octave,

$$
\exp \left[i 2 \pi\left(n+\frac{\xi}{2 \pi}\right)\right]=\exp \left[i 2 \pi \frac{\xi}{2 \pi}\right]=\exp \left[i 2 \pi \log _{2}\left(1+\frac{\delta}{2 \pi}\right)\right] \text {, }
$$

where now $0 \leq \xi / 2 \pi \leq 1$ represents a single cycle for any octave. The two cylindrical spirals (Fig. 3)

$$
\begin{aligned}
\operatorname{Sp}\left(n, \frac{\delta}{2 \pi}\right)=((x, y) & \left.=\exp \left[i 2 \pi 2^{n} \frac{\delta}{2 \pi}\right], z=2^{n}\left(1+\frac{\delta}{2 \pi}\right)\right) \\
\operatorname{Sp}\left(n, \frac{\xi}{2 \pi}\right)=((x, y) & \left.=\exp \left[i 2 \pi \log _{2}\left(1+\frac{\delta}{2 \pi}\right)\right], z=2^{n}\left(1+\frac{\delta}{2 \pi}\right)\right), \\
0 & \leq \frac{\delta}{2 \pi} \leq 1, \quad n=0,1,2, \ldots
\end{aligned}
$$

intersect at the two endpoints of each octave $n, \delta / 2 \pi=\xi / 2 \pi=0$ and $\delta / 2 \pi=\xi / 2 \pi=1$. Thus, while one loop of a logarithmic spiral defined in Eq. (3.6b) is completed, the spiral given by Eq. (3.6a) completes $n^{2}$ loops in a linear manner for each octave $n$.

A cylindrical spiral description has also been discussed in [3]. 
$\operatorname{Sp}(n, \delta / 2 \pi)$

$$
\begin{gathered}
=\left((x, y)=\exp \left[i 2 \pi 2^{n} \frac{\delta}{2 \pi}\right],\right. \\
\left.z=2^{n}(1+\delta / 2 \pi)\right) \\
0 \leq \delta / 2 \pi \leq 1, \quad n=0,1,2, \ldots
\end{gathered}
$$

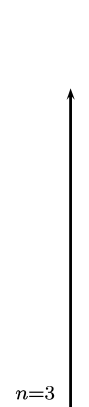

$\operatorname{Sp}(n, \xi / 2 \pi)$

$$
\begin{gathered}
=\left((x, y)=\exp \left[i 2 \pi \log _{2}\left(1+\frac{\delta}{2 \pi}\right)\right],\right. \\
\left.z=2^{n}(1+\delta / 2 \pi)\right) \\
\xi / 2 \pi=\log _{2}(1+\delta / 2 \pi) \\
0 \leq \xi / 2 \pi \leq 1, \quad n=0,1,2, \ldots
\end{gathered}
$$

\footnotetext{
(one $\xi / 2 \pi$ cycle, $0 \leq \xi / 2 \pi \leq 1$, corresponds to

$\left\{2^{n}\right.$ cycles $\delta / 2 \pi, 0 \leq \delta / 2 \pi \leq 1$,

$\left(1 / 2^{n}\right)(m-1) \leq \delta / 2 \pi \leq\left(1 / 2^{n}\right) m$,
}

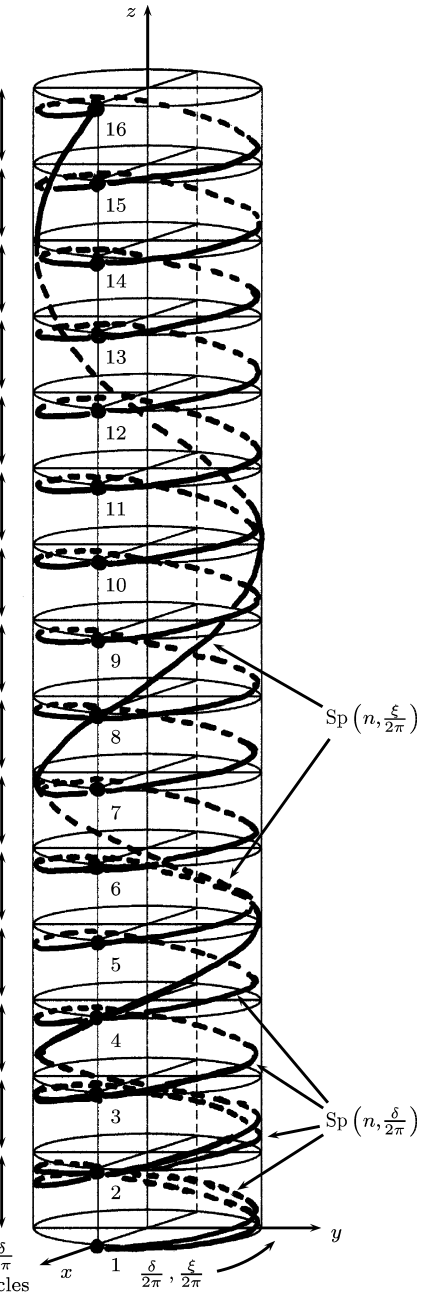

Fig. 3. The cylindrical spirals $\operatorname{Sp}(n, \delta / 2 \pi)$ and $\operatorname{Sp}(n, \xi / 2 \pi)$. These spirals show the cycle structure of the musical octave system. The parameter $\delta / 2 \pi$ is used for both spirals

An expression for the (2/1)-octave property - restricted to the plane - is given by the spiral

$$
\begin{gathered}
\operatorname{Sp}\left(\frac{\nu}{\nu_{0}}\right)=\frac{\nu}{\nu_{0}} \exp \left[i 2 \pi \frac{\delta}{2 \pi}\right]=2^{n}\left(1+\frac{\delta}{2 \pi}\right) \exp \left[i 2 \pi \frac{\delta}{2 \pi}\right], \\
n=0, \pm 1, \pm 2, \ldots, \quad 0<\frac{\delta}{2 \pi} \leq 1
\end{gathered}
$$


$\frac{\nu}{\nu_{0}}=2^{n}\left(1+\frac{\delta}{2 \pi}\right)=2^{n+\frac{\xi}{2 \pi}}$

$n$-th octave, $0 \leq \frac{\delta}{2 \pi} \leq 1$

$$
\begin{array}{ll}
\operatorname{Sp}\left(\frac{\nu}{\nu_{0}}\right)=\frac{\nu}{\nu_{0}} e^{i 2 \pi \frac{\delta}{2 \pi}}=2^{n}\left(1+\frac{\delta}{2 \pi}\right) e^{i 2 \pi \frac{\delta}{2 \pi}}, & n=0, \pm 1, \pm 2, \ldots \\
0 \leq \frac{\delta}{2 \pi} \leq 1
\end{array}
$$

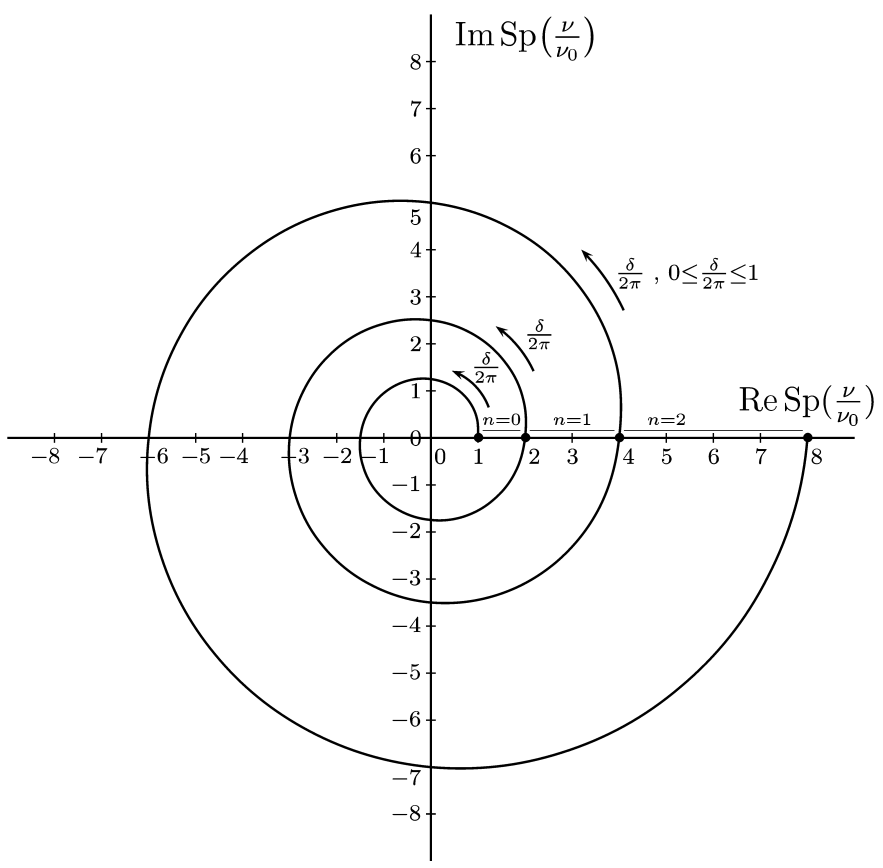

Fig. 4. This is an illustration of the (2/1)-octave property as a spiral structure in the plane. This spiral structure is the cause for the logarithmic (exponential) law for the frequencies $\nu_{\exp }$

where $n$ denotes the $n$-th "expanding loop" around the origin of the plane, Fig. 4.

\section{Derivation and Analysis}

In this section the basic results of this article are derived and discussed. 
Eq. (3.1), the basic equation for the familiar 2-based octave system, can be rewritten in the form

$$
\frac{\nu}{\nu_{0}}=2^{n}\left(1+\frac{\delta}{2 \pi}\right)=2^{n-k}\left(2^{k}-1+\left(1+2^{k} \frac{\delta}{2 \pi}\right)\right)=2^{n-k} 2^{k+(\xi / 2 \pi)},
$$

where the integer $n$ denotes the $n$-th octave, and the integer $k$ takes on the values

$$
k=0,1,2, \ldots, n .
$$

Rewriting Eq. (4.1) in the form

$$
\frac{\nu}{\nu_{0}}-2^{n-k}\left(2^{k}-1\right)=2^{n-k}\left(1+2^{k} \frac{\delta}{2 \pi}\right)=2^{n-k}\left(2^{k+(\xi / 2 \pi)}-\left(2^{k}-1\right)\right) \text {, }
$$

it is seen that the factor

$$
\left(1+2^{k} \frac{\delta}{2 \pi}\right), \quad k=0,1,2, \ldots, n
$$

in Eq. (4.2) has the property

$$
1 \leq\left(1+2^{k} \frac{\delta}{2 \pi}\right) \leq 1+2^{k}, \quad 0 \leq \frac{\delta}{2 \pi} \leq 1
$$

and represents a cycle based upon the number

$$
1+2^{k}, \quad k=0,1,2, \ldots, n .
$$

That is, cycles based upon the numbers

$$
2,3,5,9\left(=3^{2}\right), 17,33, \ldots
$$

are obtained. Considering Eq. (4.2) for the special case of $n=k$, it follows

$$
\begin{aligned}
\frac{\nu^{\prime}}{\nu_{0}} & =\frac{\nu}{\nu_{0}}-\left(2^{k}-1\right)=\left(1+2^{k} \frac{\delta}{2 \pi}\right) \\
& =2^{k+(\xi / 2 \pi)}-\left(2^{k}-1\right)=\left(1+2^{k}\right)^{\left(\xi^{\prime} / 2 \pi\right)}, \\
\frac{\xi^{\prime}}{2 \pi} & =\log _{\left(1+2^{k}\right)}\left(2^{k+(\xi / 2 \pi)}-\left(2^{k}-1\right)\right) \\
& =\log _{\left(1+2^{k}\right)}\left(2^{k}\left(1+\frac{\delta}{2 \pi}\right)-\left(2^{k}-1\right)\right) \\
& =\log _{\left(1+2^{k}\right)}\left(1+2^{k} \frac{\delta}{2 \pi}\right),
\end{aligned}
$$


with

$$
2^{(\xi / 2 \pi)}=1+\frac{\delta}{2 \pi}, \quad 0 \leq \frac{\delta}{2 \pi}, \quad \frac{\xi}{2 \pi}, \quad \frac{\xi^{\prime}}{2 \pi} \leq 1, \quad k=0,1,2, \ldots
$$

Thus, from Eq. (4.7) follows

$$
\begin{aligned}
\left(1+2^{k} \frac{\delta}{2 \pi}\right) & =\left(1+2^{k}\right)^{\left(\xi^{\prime} / 2 \pi\right)}, \quad \frac{\xi^{\prime}}{2 \pi}=\log _{\left(1+2^{k}\right)}\left(1+2^{k} \frac{\delta}{2 \pi}\right), \\
\left(1+2^{k}\right)^{w}\left(1+2^{k} \frac{\delta}{2 \pi}\right) & =\left(1+2^{k}\right)^{w+\left(\xi^{\prime} / 2 \pi\right)} \\
k & =0,1,2, \ldots, \quad 0 \leq \frac{\delta}{2 \pi}, \quad \frac{\xi^{\prime}}{2 \pi} \leq 1
\end{aligned}
$$

which is the basic law for (scaled octave) cycles based upon the numbers $\left(1+2^{k}\right), w=0, \pm 1, \pm 2, \ldots$ The value $k=0$ yields the familiar 2-based octave cycle,

$$
\begin{aligned}
\left(1+\frac{\delta}{2 \pi}\right) & =2^{\xi / 2 \pi}, \\
2^{n}\left(1+\frac{\delta}{2 \pi}\right) & =2^{n+(\xi / 2 \pi)}, \quad \frac{\xi}{2 \pi}=\log _{2}\left(1+\frac{\delta}{2 \pi}\right) .
\end{aligned}
$$

The value $k=1$ yields the 3 -based octave system

$$
\begin{gathered}
\left(1+\frac{\delta}{2 \pi}\right)=3^{\xi^{\prime} / 2 \pi}, \\
3^{m}\left(1+2 \frac{\delta}{2 \pi}\right)=3^{m+\left(\xi^{\prime} / 2 \pi\right)}, \\
\frac{\xi^{\prime}}{2 \pi}=\frac{1}{\log _{2} 3} \log _{2}\left(2^{1+(\xi / 2 \pi)}-1\right)=\log _{3}\left(1+2 \frac{\delta}{2 \pi}\right), \\
3^{n} \nu_{0} \leq 3^{m} \nu_{0}\left(1+2 \frac{\delta}{2 \pi}\right) \leq 3^{m+1} \nu_{0}, \\
m=0,1,2, \ldots, \quad 0 \leq \frac{\delta}{2 \pi}, \quad \frac{\xi}{2 \pi}, \quad \frac{\xi^{\prime}}{2 \pi} \leq 1
\end{gathered}
$$

and value $k=2$ yields the 5 -based octave system

$$
\left(1+2^{2} \frac{\delta}{2 \pi}\right)=5^{\xi^{\prime \prime} / 2 \pi},
$$




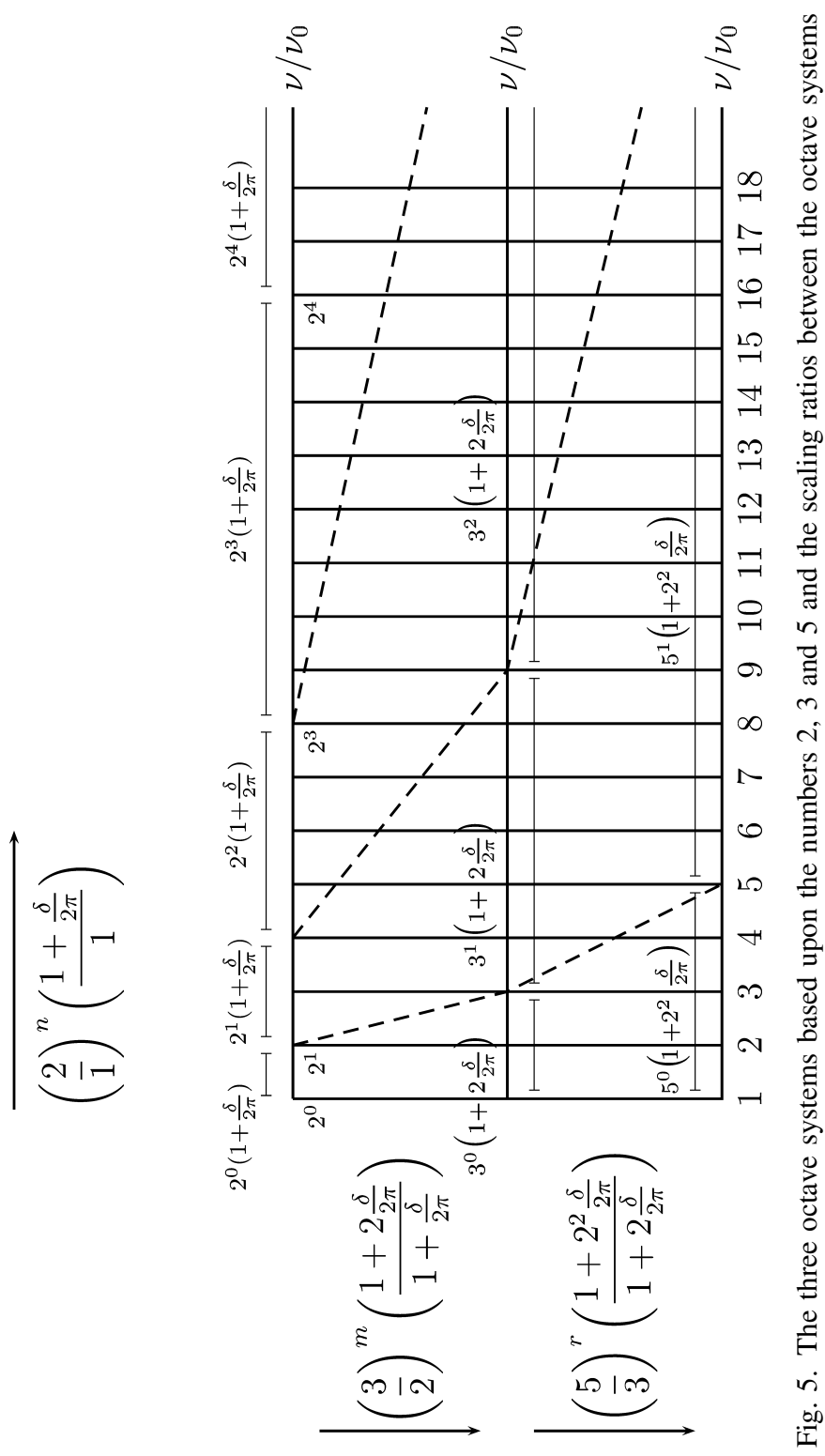




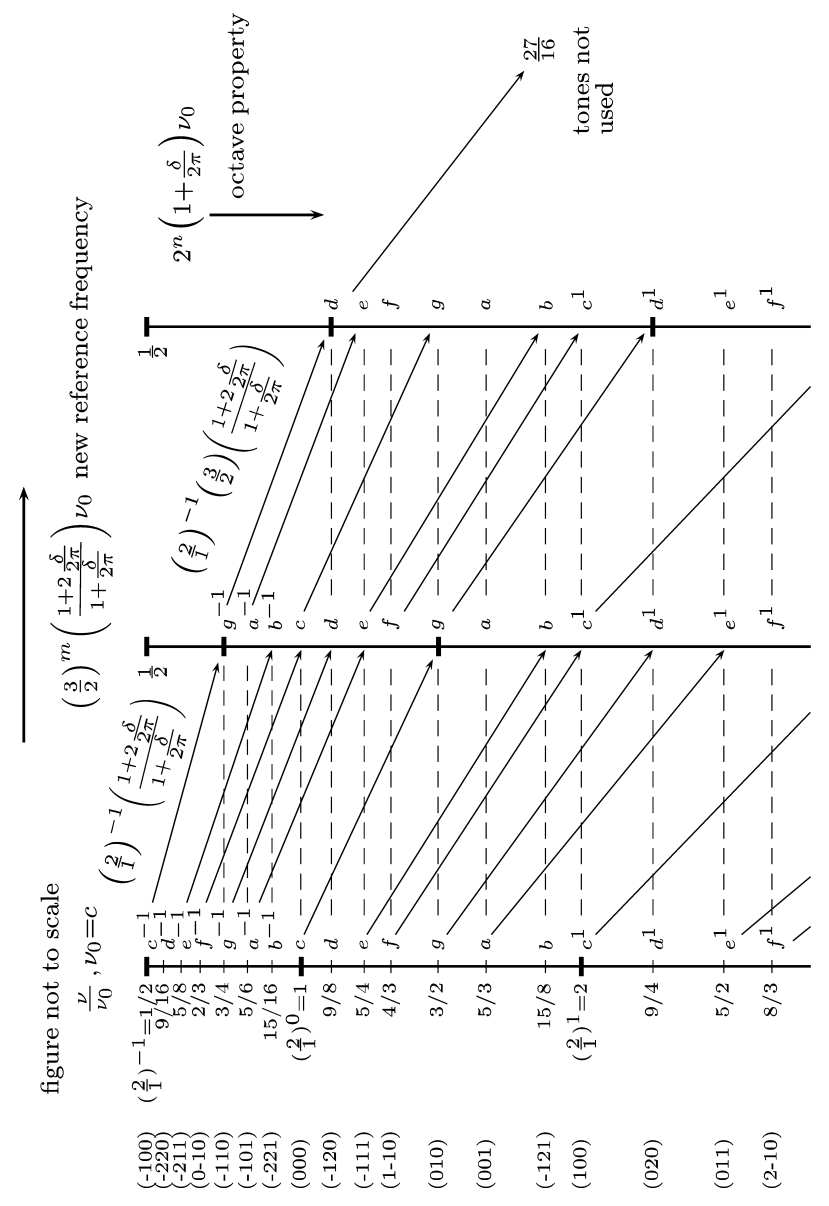



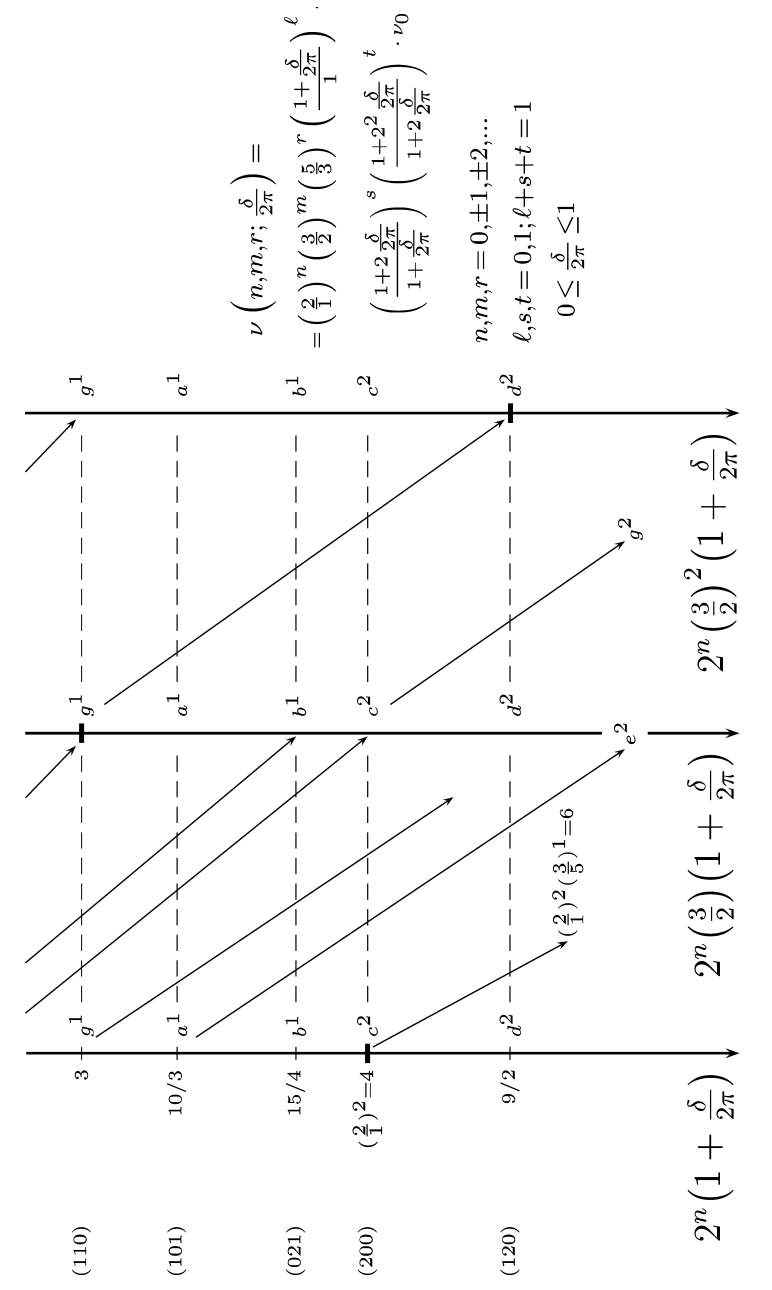

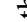

$\stackrel{2}{0} \approx$

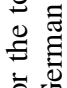

ฮัญ

总

0
0

$\doteq 0$

范

๑ิ

竞:

$\stackrel{x}{i}$

(⿸\zh14⿰丿⿱丄𠃍)

范

b 키

E

in

完完

(1)

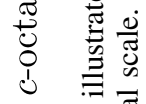

:

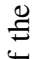

㱏

6

宔 


$$
\begin{gathered}
5^{r}\left(1+2^{2} \frac{\delta}{2 \pi}\right)=5^{r+\left(\xi^{\prime \prime} / 2 \pi\right)}, \\
5^{r} \nu_{0} \leq 5^{r}\left(1+2^{2} \frac{\delta}{2 \pi}\right) \leq 5^{r+1} \nu_{0}, \\
\frac{\xi^{\prime \prime}}{2 \pi}=\log _{5}\left(2^{2+(\xi / 2 \pi)}-3\right)=\log _{5}\left(1+2^{2} \frac{\delta}{2 \pi}\right), \\
r=0,1,2, \ldots, \quad 0 \leq \frac{\delta}{2 \pi}, \quad \frac{\xi}{2 \pi}, \quad \frac{\xi^{\prime \prime}}{2 \pi} \leq 1,
\end{gathered}
$$

Fig. 5 and Fig. 6.

Higher scaling factors appear not to contribute, possibly because the scaling becomes too large, involving too many ( $\left.\frac{2}{1}\right)$-octaves (see Fig. 3).

Considering Eq. (4.2) for the special values $n=1, k=0$ and $n=2, k=1$, it can be seen that the numbers 4 and 6 are in fact composite numbers, namely 2.2 and 2.3 . That is, they are built up from the basic set $(2,3,5)$

$$
\begin{gathered}
n=1, k=0: \quad \frac{\nu}{\nu_{0}}=2 \cdot\left(1+\frac{\delta}{2 \pi}\right)=2 \cdot 2^{(\xi / 2 \pi)}, \\
\text { for } \quad \frac{\delta}{2 \pi}=\frac{\xi}{2 \pi}=1
\end{gathered}
$$

and

$$
\begin{gathered}
n=2, k=3: \quad \frac{\nu}{\nu_{0}}-2=2 \cdot\left(1+2 \frac{\delta}{2 \pi}\right)=2\left(2^{1+(\xi / 2 \pi)}-1\right), \\
\text { for } \quad \frac{\delta}{2 \pi}=\frac{\xi}{2 \pi}=1 .
\end{gathered}
$$

The 2-based, 3-based and 5-based octave systems are called octave systems since they carry the octave system properties of the standard musical octave system, which is based upon 2. This property is inherited from the original 2-based octave system since the 3-based and 5-based octave systems are obtained by means of similarity (scaling) transformations which do not alter the musical properties (ratios). This is analogous to the manner by which the $n \neq 0,2$-based octaves, inherit the properties from the basic $n=0$ octave, $\nu_{0} \leq \nu \leq 2 \nu_{0}$, by means of the scaling transformation

$$
\left(1+\frac{\delta}{2 \pi}\right), \quad 0 \leq \frac{\delta}{2 \pi} \leq 1 .
$$


For example, scaling the basic octave $n=0$ one obtains

$$
\left(1+\frac{\delta}{2 \pi}\right) \nu_{0} \leq\left(1+\frac{\delta}{2 \pi}\right) \nu \leq\left(1+\frac{\delta}{2 \pi}\right) 2 \nu_{0}, \quad 0 \leq \frac{\delta}{2 \pi} \leq 1,
$$

which yields for $\delta / 2 \pi=1$ the scaled octave $n=1$,

$$
2 \nu_{0} \leq \nu^{\prime}=2 \nu \leq 2^{2} \nu_{0}
$$

that is, the next octave (together with its inherited musical properties).

The scaling (similarity) transformation which carries the $\left(1+2^{k}(\delta / 2 \pi)\right)$-based octave into the $\left(1+2^{k+1}(\delta / 2 \pi)\right)$-based octave is given by

$$
\frac{\left(1+2^{k+1}(\delta / 2 \pi)\right)}{\left(1+2^{k}(\delta / 2 \pi)\right)}
$$

which for $\delta / 2 \pi=1$ yields the scaling ratios

$$
\frac{2}{1}, \quad \frac{\left(1+2^{k+1}\right)}{\left(1+2^{k}\right)}, \quad k=0,1,2,3, \ldots
$$

The combined octave system consists of the product of the three octave systems given by the product of the scalings

$$
(n, m, r) \equiv\left(\frac{2}{1}\right)^{n}\left(\frac{3}{2}\right)^{m}\left(\frac{5}{3}\right)^{r} \text {. }
$$

It follows that

$$
(n, m, r)=(100)^{n}(010)^{m}(001)^{r},
$$

where

$$
(100)^{n},(010)^{m} \text { and }(001)^{r}, \quad n, m, r=0, \pm 1, \pm 2
$$

represent the $\left(\frac{2}{1}\right)$-based, $\left(\frac{3}{2}\right)$-based and $\left(\frac{5}{3}\right)$-based octave systems with respect to a frequency (tone) $\nu_{0}$,

$$
\begin{aligned}
\nu=(100)^{n} \nu_{0}, \quad \nu & =(010)^{m} \nu_{0}, \quad \nu=(001)^{r} \nu_{0}, \\
n, m, r & =0, \pm 1, \pm 2, \ldots
\end{aligned}
$$

The three elements

$$
\text { (100), (010), (001) }
$$

can be considered to be linearly independent orthogonal basis elements of a 3-dimensional space. Then the correspondence

$$
(n, m, r) \leftrightarrow n(100)+m(010)+r(001) \equiv \ell(n, m, r)
$$

maps the elements $(n, m, r)$ upon the lattice points $\ell(n, m, r)$. This map yields a (scaled) lattice for the system of musical tones. For 
simplicity the lattice points $\ell(n, m, r)$ will henceforth also be denoted by $(n, m, r)$ as there is little chance for confusion of the two meanings in the context of this article (Sect. 7).

The definition Eq. (4.17) also shows that the multiplication of two frequency ratios (numbers) is equivalent to the vector addition of the exponents. Both operations will be used interchangeably.

The numerical condition

$$
A \leq(n m r) \leq B
$$

defines the lattice points $(n, m, r)$ for which the musical ratio, with respect to a chosen reference frequency $\nu_{0}$, falls within the parameter values $A$ and $B$. Thus, for $A=1$ and $B=2$, the condition

$$
\left(\frac{2}{1}\right)^{0}=1 \leq(n 00) \leq\left(\frac{2}{1}\right)^{1}=2
$$

implies that $n=0,1$ only. That is, the basic $n=0$, 2-based octave, with the lattice points (tones) $\nu_{0}(=c)$ and $2 \nu_{0}\left(=c^{1}\right)$ is obtained. Similarly

$$
\left(\frac{3}{2}\right)^{0}=1 \leq(0 m 0) \leq\left(\frac{3}{2}\right)^{1} \text { and }\left(\frac{5}{3}\right)^{0} \leq(00 r) \leq\left(\frac{5}{3}\right)^{1}
$$

yield $m=0,1$ and $r=0,1$, the basic $m=0$ and $r=0,3$-based and 5-based octaves corresponding to the tones $c, g$ and $c, a$ of the lattice.

The numerical condition

$$
1 \leq(n, m, 0) \leq 2
$$

is equivalent to the condition

$$
2^{m-n} \leq 3^{m} \leq 2^{m-n+1} .
$$

This condition leads to the extended Pythagorean scale, Sect. 7 and Fig. 9.

The general condition

$$
1 \leq(n, m, r) \leq 2
$$

can be reformulated as

$$
2^{a} \leq 3^{b} 5^{c} \leq 2^{a+1}
$$

with

$$
\begin{aligned}
& a=m-n, \\
& b=m-r, \\
& c=r,
\end{aligned}
$$


corresponding to the elements/lattice points

$$
(n=b+c-a, m=b+c, r=c) .
$$

The various lattices shown in the figures can be derived from Eq. (4.23). They define, via their lattice points, the intrinsic musical properties of a musical system. External requirements, like the requirement of "equal spacing" between tones for mechanical instruments of western culture $(\sqrt[12]{2})$, will cause disturbances of the symmetry of the musical lattice, i.e. will "break its symmetry."

\section{Results and Summary}

The musical tones, defined by their frequency ratios, can formally be looked upon as lattice points (vectors) in a 3-dimensional lattice space. The lattice lines represent - in a formal way - "rescaled octave systems". That is, the lattice points along the $\left(\frac{2}{1}\right)$-octave line correspond to the octave tones $c^{n}, n=0, \pm 1, \pm 2, \ldots$ The lattice points along the rescaled $\left(\frac{3}{2}\right)$-octave line correspond to the tones with frequency ratios $\left(\frac{3}{2}\right)^{m}, m=0, \pm 1, \pm 2, \ldots$ The lattice points along the rescaled $\left(\frac{5}{3}\right)$-octave line correspond to the tones with frequency ratios $\left(\frac{5}{3}\right)^{r}, r=0, \pm 1, \pm 2, \ldots$ All tones of the 3-dimensional lattice system are then given by the frequency ratios $\left(\frac{2}{1}\right)^{n}\left(\frac{3}{2}\right)^{m}\left(\frac{5}{3}\right)^{r}-$ the so-called numerical values of the lattice points $(n, m, r)$. These numerical values represent a map from the lattice structure onto the line of frequency ratios - the standard theory of musical tones.

A geometrical picture for the 3-dimensional musical tone system is thus given by

$$
\begin{aligned}
\nu\left(n, m, r ; \frac{\delta}{2 \pi}\right)= & \left(\frac{2}{1}\right)^{n}\left(\frac{3}{2}\right)^{m}\left(\frac{5}{3}\right)^{r} \\
& \times\left(\frac{1+(\delta / 2 \pi)}{1}\right)^{\ell}\left(\frac{1+2(\delta / 2 \pi)}{1+(\delta / 2 \pi)}\right)^{s} \\
& \times\left(\frac{1+2^{2}(\delta / 2 \pi)}{1+2(\delta / 2 \pi)}\right)^{t} \cdot \nu_{0},
\end{aligned}
$$

$n, m, r=0, \pm 1, \pm 2, \ldots, \quad \ell, s, t=0,1, \quad \ell+s+t=1, \quad 0 \leq \frac{\delta}{2 \pi} \leq 1$, whereby the frequency ratios

$$
\nu\left(n, m, r ; \frac{\delta}{2 \pi}=0\right) / \nu_{0}=\left(\frac{2}{1}\right)^{n}\left(\frac{3}{2}\right)^{m}\left(\frac{5}{3}\right)^{r} \equiv(n, m, r)
$$


correspond to the musical tones of the system, namely the octave system, having the property $\delta / 2 \pi=\xi / 2 \pi=0,1$.

The musical system (5.1) rests upon three octave systems, namely the systems

$$
\begin{gathered}
\begin{aligned}
\nu\left(n, 0,0 ; \frac{\delta}{2 \pi}\right)=2^{n}\left(1+\frac{\delta}{2 \pi}\right) \nu_{0}, \quad n=0, \pm 1, \pm 2, \ldots \\
(2 \text {-based octave system }),
\end{aligned} \\
\nu\left(0, m, 0 ; \frac{\delta}{2 \pi}\right)=3^{m}\left(\begin{array}{r}
\left.1+2 \frac{\delta}{2 \pi}\right) \nu_{0}, \quad m=0, \pm 1, \pm 2, \ldots \\
(3 \text {-based octave system }),
\end{array}\right. \\
\nu\left(0,0, r ; \frac{\delta}{2 \pi}\right)=5^{r}\left(\begin{array}{r}
\left.1+2^{2} \frac{\delta}{2 \pi}\right) \nu_{0}, \quad r=0, \pm 1, \pm 2, \ldots \\
(5 \text {-based octave system }), \\
0 \leq \frac{\delta}{2 \pi} \leq 1 .
\end{array}\right.
\end{gathered}
$$

The relationship between the two bases used in Eq. (5.1) and Eq. (5.3) is given by

$$
\begin{array}{rlrl}
\left(\frac{2}{1}\right)^{n}\left(\frac{3}{2}\right)^{m}\left(\frac{5}{3}\right)^{r} & =2^{n^{\prime}} 3^{m^{\prime}} 5^{r^{\prime}}, \\
n^{\prime} & =n-m, \quad n & =n^{\prime}+m^{\prime}+r^{\prime}, \\
m^{\prime} & =m-r, & m & =m^{\prime}+r^{\prime}, \\
r^{\prime} & =r, & r & =r^{\prime} .
\end{array}
$$

The scaling factors between these octave systems are given by

$$
\begin{array}{cc}
\frac{\nu(n, 0,0 ; \delta / 2 \pi)}{\nu_{0}}=\left(\frac{2}{1}\right)^{n} \frac{(1+(\delta / 2 \pi))}{1}, & n=0, \pm 1, \pm 2, \ldots \\
\frac{\nu(0, m, 0 ; \delta / 2 \pi)}{\nu(m, 0,0 ; \delta / 2 \pi)}=\left(\frac{3}{2}\right)^{m} \frac{(1+2(\delta / 2 \pi))}{(1+(\delta / 2 \pi))}, & m=0, \pm 1, \pm 2, \ldots \\
\frac{\nu(0,0, r ; \delta / 2 \pi)}{\nu(0, r, 0 ; \delta / 2 \pi)}=\left(\frac{5}{3}\right)^{r} \frac{\left(1+2^{2}(\delta / 2 \pi)\right)}{(1+2(\delta / 2 \pi))}, & r=0, \pm 1, \pm 2, \ldots \\
0 \leq \frac{\delta}{2 \pi} \leq 1 &
\end{array}
$$

with

$$
\nu_{0}=\nu(0,0,0 ; 0)
$$

the reference frequency. 
For $n=0, m=0, r=0, \delta / 2 \pi=0$, the three ratios

$$
\left(\frac{2}{1}\right), \quad\left(\frac{3}{2}\right) \text { and }\left(\frac{5}{3}\right)
$$

are obtained.

The composite musical octave system (5.1) is given by the product of the three $(\delta / 2 \pi)$-dependent scaling factors (5.5). The scaling factor $\delta / 2 \pi$ can be changed for only one of the three scalings (5.5) at a time resulting in the condition $\ell, s, t=0,1$, with $\ell+s+t=1$.

The ratios

$$
\frac{\nu(n, m, r ; \delta / 2 \pi=0)}{\nu_{0}}=\left(\frac{2}{1}\right)^{n}\left(\frac{3}{2}\right)^{m}\left(\frac{5}{3}\right)^{r} \equiv(n, m, r)
$$

correspond to the musical tones $(\delta / 2 \pi=\xi / 2 \pi=0)$ of the musical lattice system with respect to the reference tone $\nu_{0}$. These frequency ratios can be represented as lattice points of a 3-dimensional lattice with the distances between the lattice points along the three axes scaled by the factors $\left(\frac{2}{1}\right)^{n},\left(\frac{3}{2}\right)^{m},\left(\frac{5}{3}\right)^{r}$, respectively, Fig. 12 and Fig. 15 .

In turn, for fixed values $n, m, r$, the frequency $\nu_{0}^{\prime}$,

$$
\nu_{0}^{\prime}=\nu\left(n, m, r ; \frac{\delta}{2 \pi}=0\right) \nu_{0}=\left(\frac{2}{1}\right)^{n}\left(\frac{3}{2}\right)^{m}\left(\frac{5}{3}\right)^{r} \nu_{0},
$$

can be chosen as reference frequency for a musical lattice system with respect to the tone $\nu_{0}^{\prime}$. Then

$$
\begin{aligned}
\nu\left(n^{\prime}, m^{\prime}, r^{\prime} ; \frac{\delta}{2 \pi}\right)= & \left(\frac{2}{1}\right)^{n^{\prime}}\left(\frac{3}{2}\right)^{m^{\prime}}\left(\frac{5}{3}\right)^{r^{\prime}} \\
& \times\left(\frac{1+(\delta / 2 \pi)}{1}\right)^{\ell}\left(\frac{1+2(\delta / 2 \pi)}{1+(\delta / 2 \pi)}\right)^{s}\left(\frac{1+2^{2}(\delta / 2 \pi)}{1+2(\delta / 2 \pi)}\right)^{t} \cdot \nu_{0}^{\prime}
\end{aligned}
$$

is an equivalent musical system with respect to the reference tone $\nu_{0}^{\prime}$. Its musical tones, with respect to $\nu_{0}^{\prime}$ and with respect to $\nu_{0}$, are given by the lattice points

$$
\begin{aligned}
\frac{\nu\left(n^{\prime}, m^{\prime}, r^{\prime} ; \delta / 2 \pi=0\right)}{\nu_{0}^{\prime}} & =\left(n^{\prime}, m^{\prime}, r^{\prime}\right), \text { with respect to } \nu_{0}^{\prime}, \\
& =\frac{\nu\left(n^{\prime}-n, m^{\prime}-m, r^{\prime}-r ; \delta / 2 \pi=0\right)}{\nu_{0}} \\
& =\left(n^{\prime}-n, m^{\prime}-m, r^{\prime}-r\right), \text { with respect to } \nu_{0} .
\end{aligned}
$$


Thus the ratio of the tones of the $\nu_{0}^{\prime}$-based octave system with respect to the tones of the $\nu_{0}$-based octave system

$$
\frac{\nu_{0}^{\prime}}{\nu_{0}}=\frac{\nu\left(n^{\prime}, m^{\prime}, r^{\prime} ; 0\right)}{\nu\left(n^{\prime}-n, m^{\prime}-m, r^{\prime}-r ; 0\right)}=\left(\frac{2}{1}\right)^{n}\left(\frac{3}{2}\right)^{m}\left(\frac{5}{3}\right)^{r}
$$

can be looked upon as the defining ratio of the musical tones which correspond to the two frequencies $\nu_{0}^{\prime}$ and $\nu_{0}$. That is, each musical tone has associated with it its own lattice octave system with all its tones defined by $(\delta / 2 \pi=\xi / 2 \pi=0,1)$, and the musical ratio $\nu_{0}^{\prime} / \nu_{0}$ for tones is a ratio between their associated lattice octave systems.

The interval factors between two distinct tones $(n, m, r)$ and $\left(n^{\prime}, m^{\prime}, r^{\prime}\right)$ of a given musical system with respect to a fixed reference tone $\nu_{0}$ is given by

$$
(n, m, r)-\left(n^{\prime}, m^{\prime}, r^{\prime}\right)=\left(n-n^{\prime}, m-m^{\prime}, r-r^{\prime}\right)
$$

and it holds for the tones of a musical scale that

$$
\sum(n, m, r)=(N, 0,0)
$$

where the sum is over all tones $(n, m, r)$ of the scale and $N$ is the number of 2-octaves of the scale.

Most of the musical tones encountered in this article can be found in the list of tones given in [4]. The basis used in [4] is

$$
\begin{array}{rll}
c^{1} / c=2 / 1 & =(1,0,0) & =O \text { (Octave) } \\
e / c=5 / 4 & =(-1,1,1) & =T \text { (Terz) } \\
g / c=3 / 2 & =(0,1,0) & =Q \text { (Quint) } .
\end{array}
$$

The basis used in this article is

$$
\begin{array}{rll}
c^{1} / c=2 / 1 & =(1,0,0) & =O \text { (Octave) } \\
g / c=3 / 2 & =(0,1,0) & =Q \text { (Quint) } \\
a / c=5 / 3 & =(0,0,1) & =S \text { (Sixth) }
\end{array}
$$

The two bases are mathematically equivalent. They are related to each other by

$$
\begin{aligned}
& T=e / c=5 / 4=Q S / O=(-1,1,1), \\
& S=a / c=5 / 3=O T / Q=(0,0,1) .
\end{aligned}
$$

\section{Examples and Applications}

In the following, various musical scales are listed in order to illustrate the results obtained. For ease of comparison, both the standard fractions for the tones and intervals (factors) and their internal structure $(n, m, r)$ is given. Note that English $b^{b}$ and $b$ correspond to German $b$ 
and $h$, respectively, and that $(n m r) /\left(n^{\prime} m^{\prime} r^{\prime}\right)=\left(n-n^{\prime}, m-m^{\prime}, r-r^{\prime}\right)$ are the interval factors.

\subsection{The 2-Based (Standard) Octave System}

The 2-based (standard) octave system is fully characterized by the subset $\left\{(n, 0,0)=\left(\frac{2}{1}\right)^{n} ; n=0, \pm 1, \pm 2, \ldots\right\}$

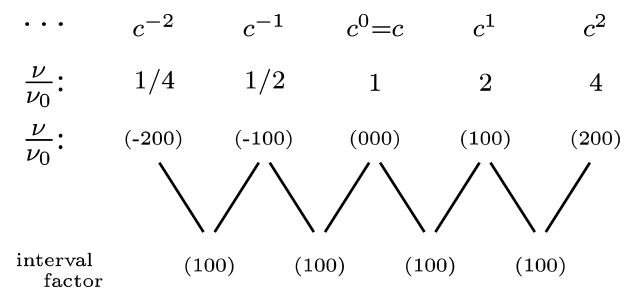

The ancient Greek Lyre and the Pythagorean musical scale are based upon the subset $\{(n, m, 0) ; n, m=0, \pm 1, \pm 2, \ldots\}$.

\subsection{The Ancient Greek Lyre}

$$
\begin{aligned}
& \left\{(n, m, 0)=\left(\frac{2}{1}\right)^{n}\left(\frac{3}{2}\right)^{m} ; n, m=0, \pm 1, \pm 2, \ldots\right\}
\end{aligned}
$$

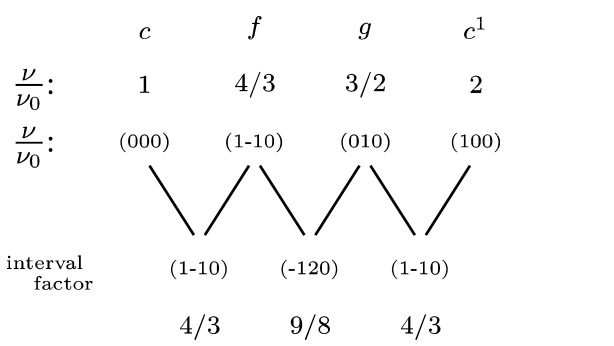

\subsection{Pythagorean Diatonic Musical Scale}

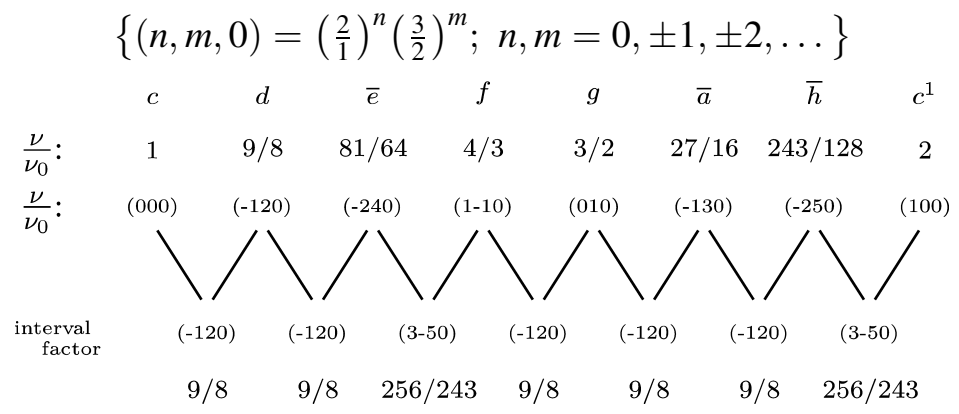

Syntonic comma: $\frac{80}{81}=\left(\frac{2}{1}\right)^{1}\left(\frac{3}{2}\right)^{-3}\left(\frac{5}{3}\right)^{1}$ 


\subsection{The Natural Diatonic Scale}

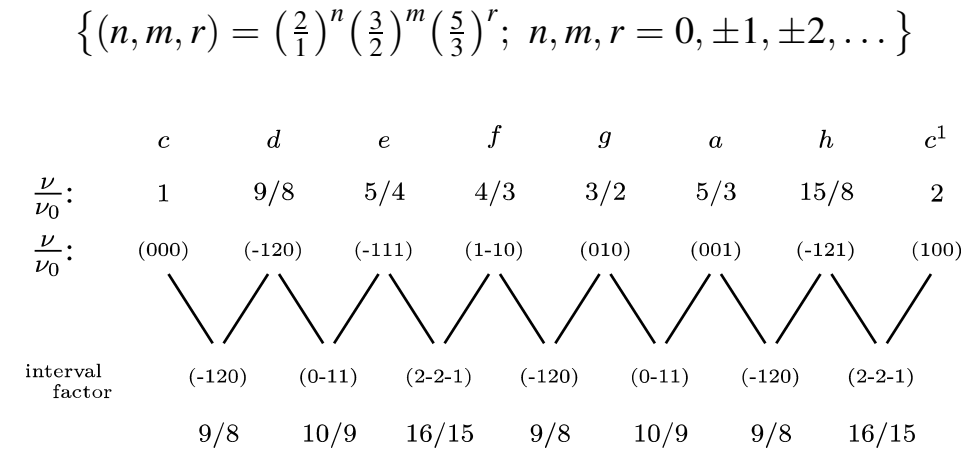

6.5. The Chromatic Scale, Major

$\left\{(n, m, r)=\left(\frac{2}{1}\right)^{n}\left(\frac{3}{2}\right)^{m}\left(\frac{5}{3}\right)^{r} ; n, m, r=0, \pm 1, \pm 2, \ldots\right\}$

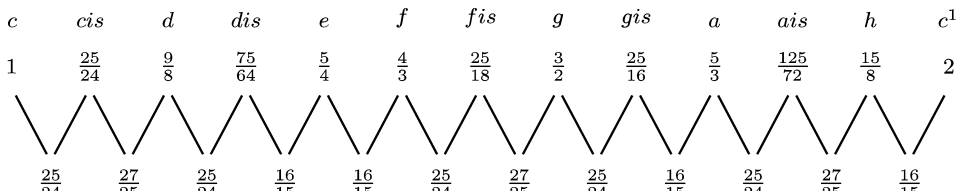

$\begin{array}{lllllllllllll}(000) & (-212) & (-120) & (-332) & (-111) & (1-10) & (-102) & (010) & (-222) & (001) & (-213) & (-121) & (100)\end{array}$

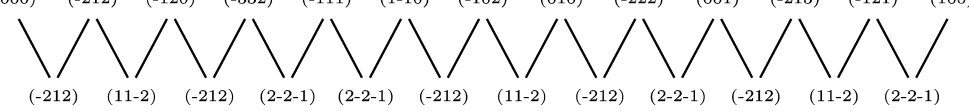

\subsection{The Chromatic Scale, Minor}

$\left\{(n, m, r)=\left(\frac{2}{1}\right)^{n}\left(\frac{3}{2}\right)^{m}\left(\frac{5}{3}\right)^{r} ; n, m, r=0, \pm 1, \pm 2, \ldots\right\}$

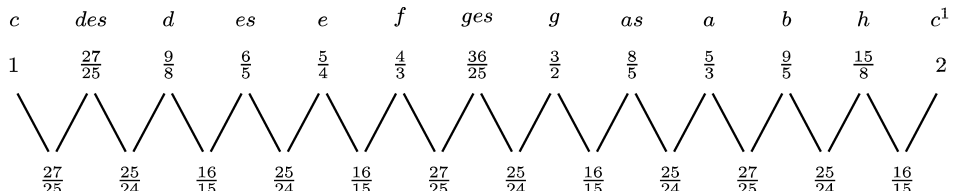

$\begin{array}{lllllllllllll}(000) & (11-2) & (-120) & (10-1) & (-111) & (1-10) & (20-2) & (010) & (2-1-1) & (001) & (11-1) & (-121) & (100)\end{array}$

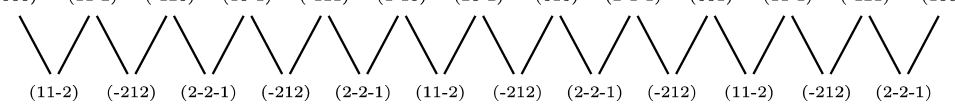




\subsection{NOH (Theatre, Dance) [2]}

$\mathrm{NOH}$, tone system for singing with respect to tone $c$. $\left\{(n, m, r)=\left(\frac{2}{1}\right)^{n}\left(\frac{3}{2}\right)^{m}\left(\frac{5}{3}\right)^{r} ; n, m, r=0, \pm 1, \pm 2, \ldots\right\}$

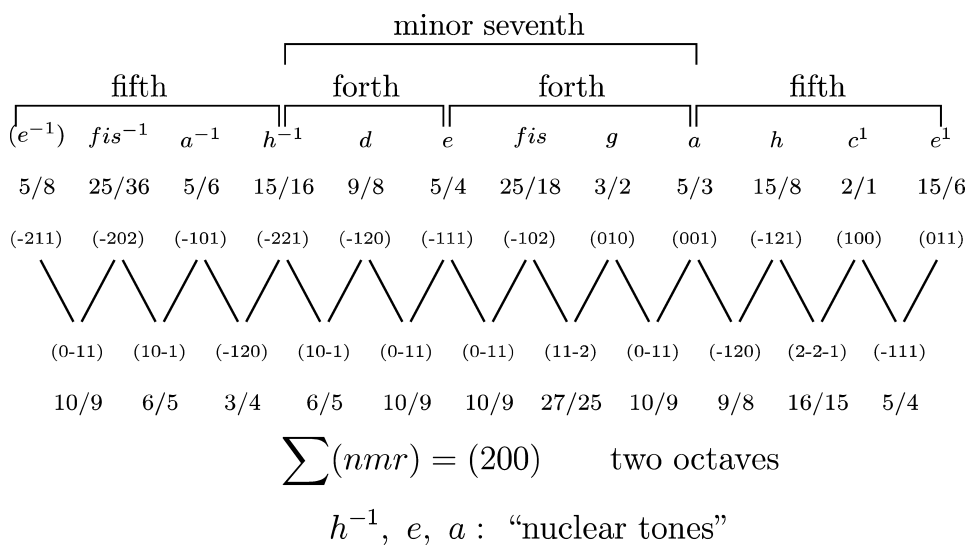

\subsection{NOH [2]}

Transverse bamboo flute, Noh-kan

$\left\{(n, m, r)=\left(\frac{2}{1}\right)^{n}\left(\frac{3}{2}\right)^{m}\left(\frac{5}{3}\right)^{r} ; n, m, r=0, \pm 1, \pm 2, \ldots\right\}$

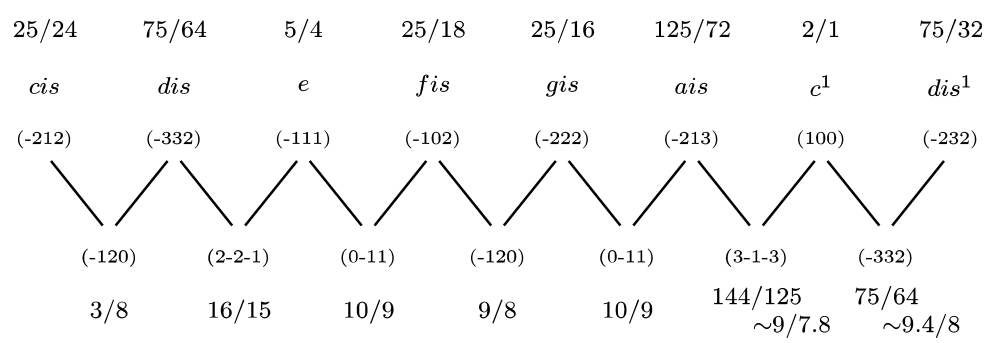

\section{The Scaled Lattices}

In this section the scaled lattices and their musical contents are listed for those musical scales which were discussed in this article. These lattices represent the internal structure of the musical tones and of the interval factors associated with them. 


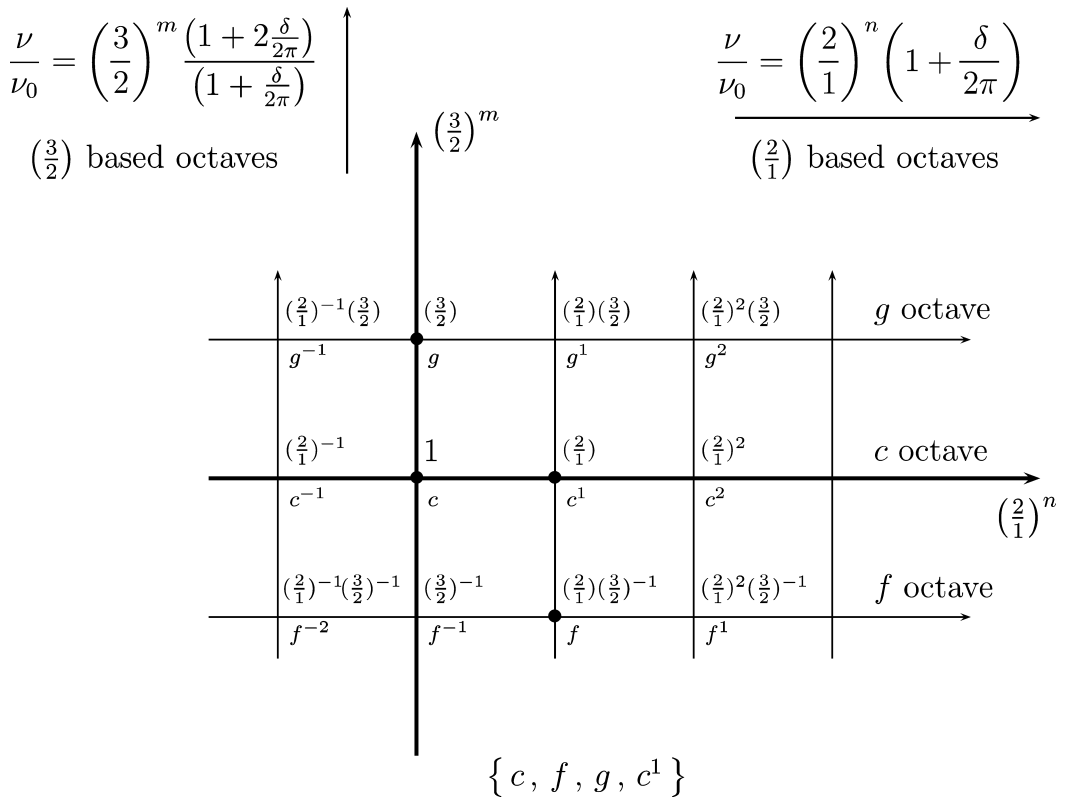

Fig. 7. It shows part of the $(n, m, 0)$ lattice with the four tones of the ancient Greek Lyre

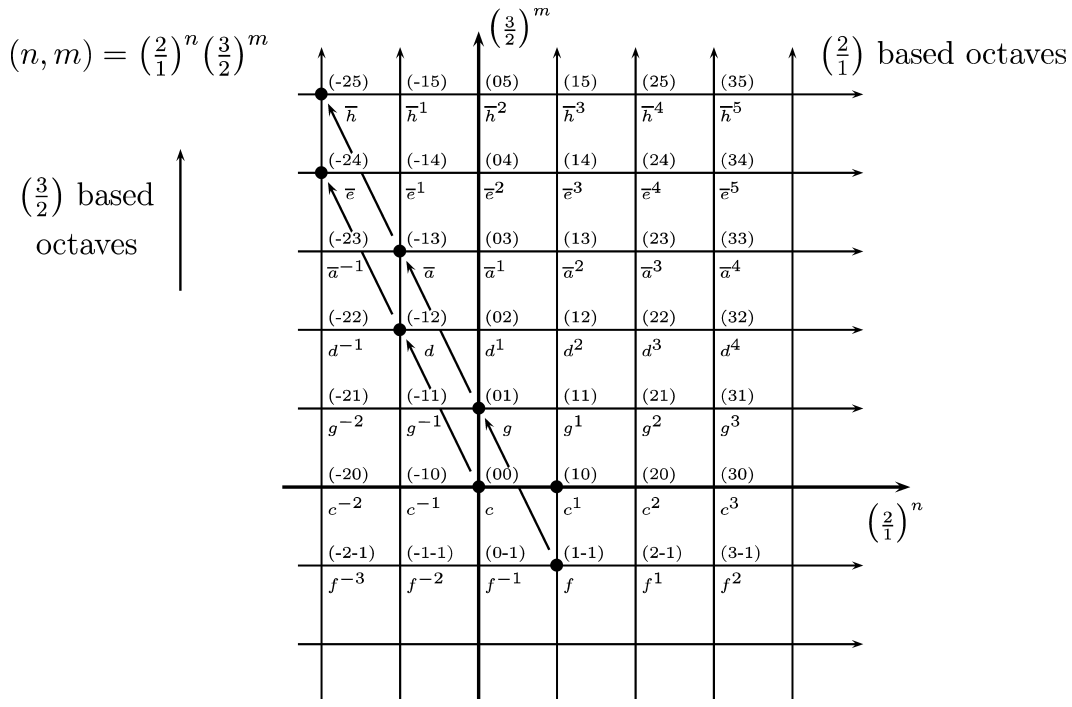

Fig. 8. It shows a larger portion of the $(n, m, 0)$ lattice with the tones of the diatonic Pythagorean scale indicated in it 


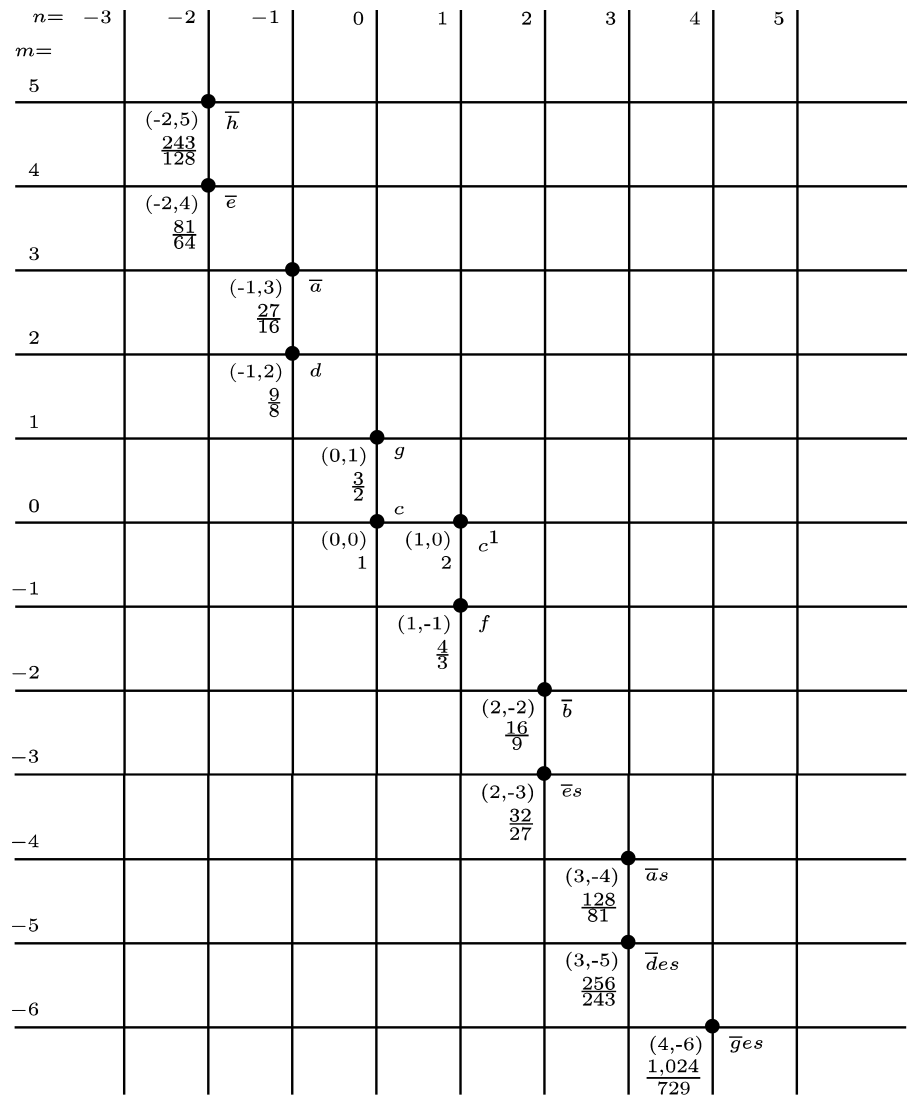

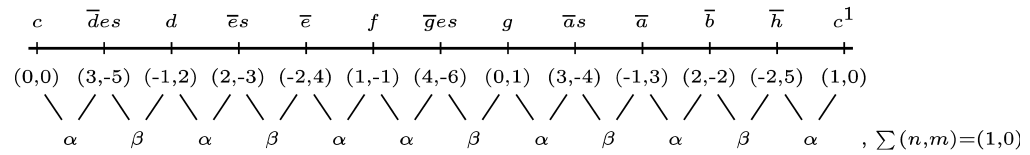

Interval vectors/factors:

$$
\begin{aligned}
& \alpha=(3,-5) ; 256 / 243 \\
& \beta=(-4,7) ; 2,187 / 2,048, \quad \beta \alpha^{-1}=(-7,12) \text { the Pythagorean comma, } \\
& \alpha^{7} \beta^{5}=\left((2 / 1)^{3}(3 / 2)^{-5}\right)^{7}\left((2 / 1)^{-4}(3 / 2)^{7}\right)^{5}=2
\end{aligned}
$$

Fig. 9. It shows an extended Pythagorean scale consisting of 12 tones 


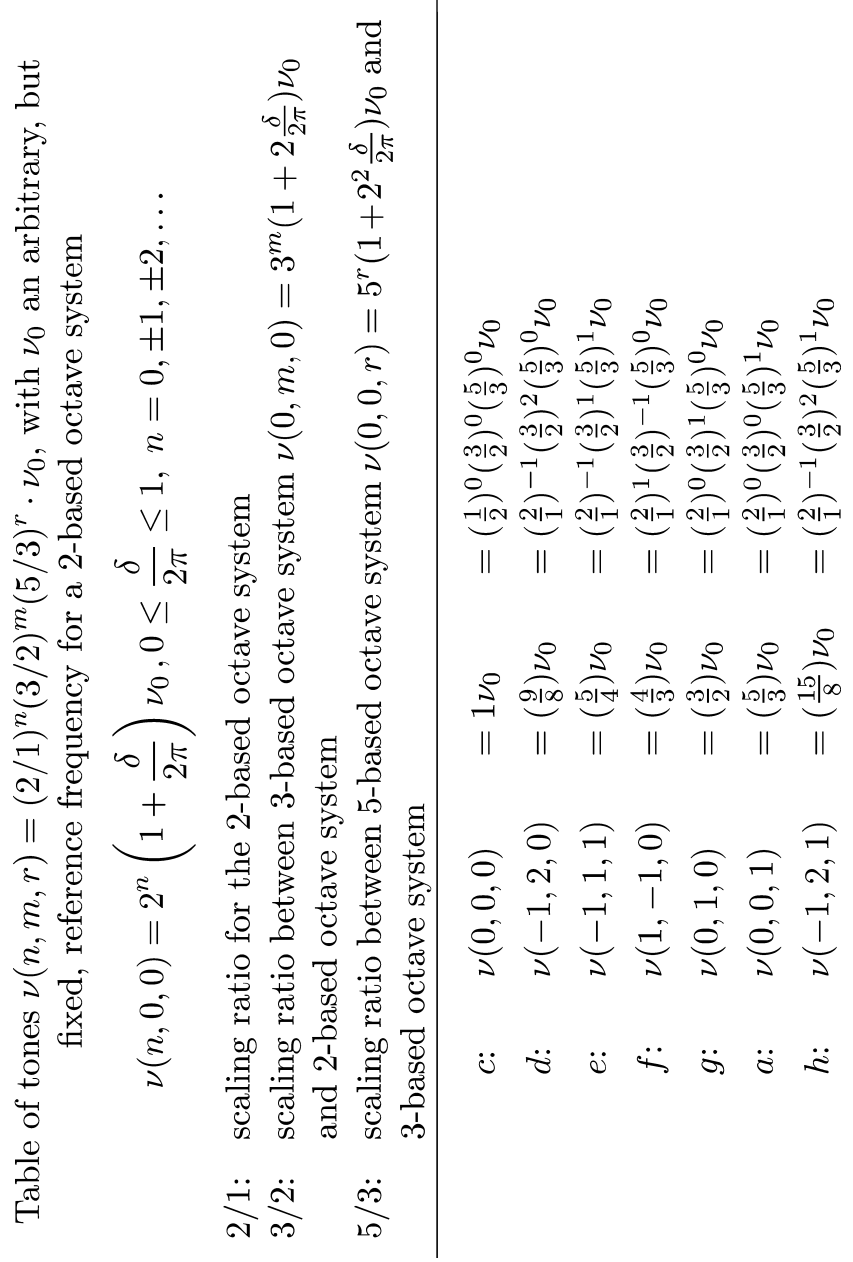




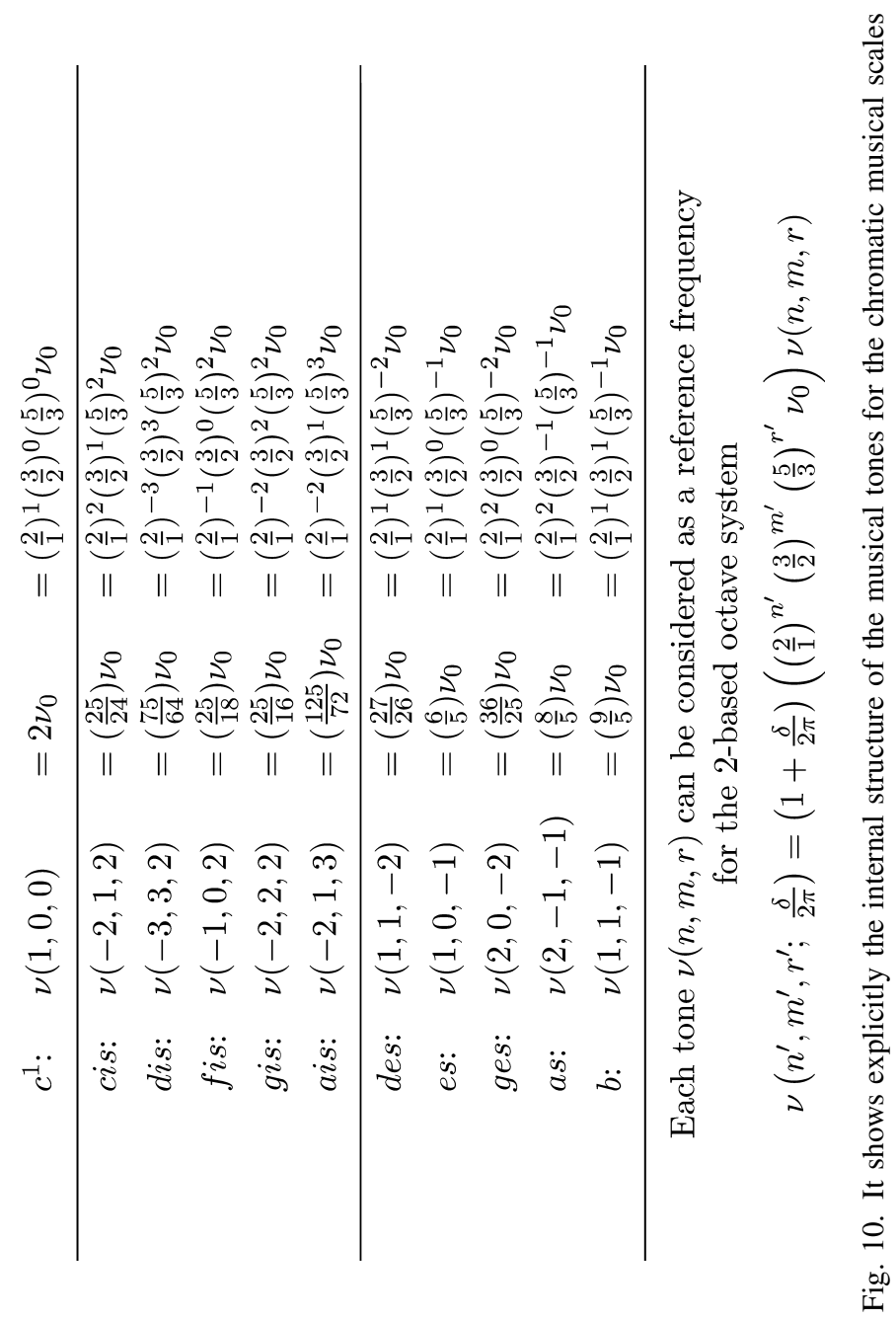




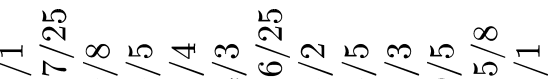

舟 


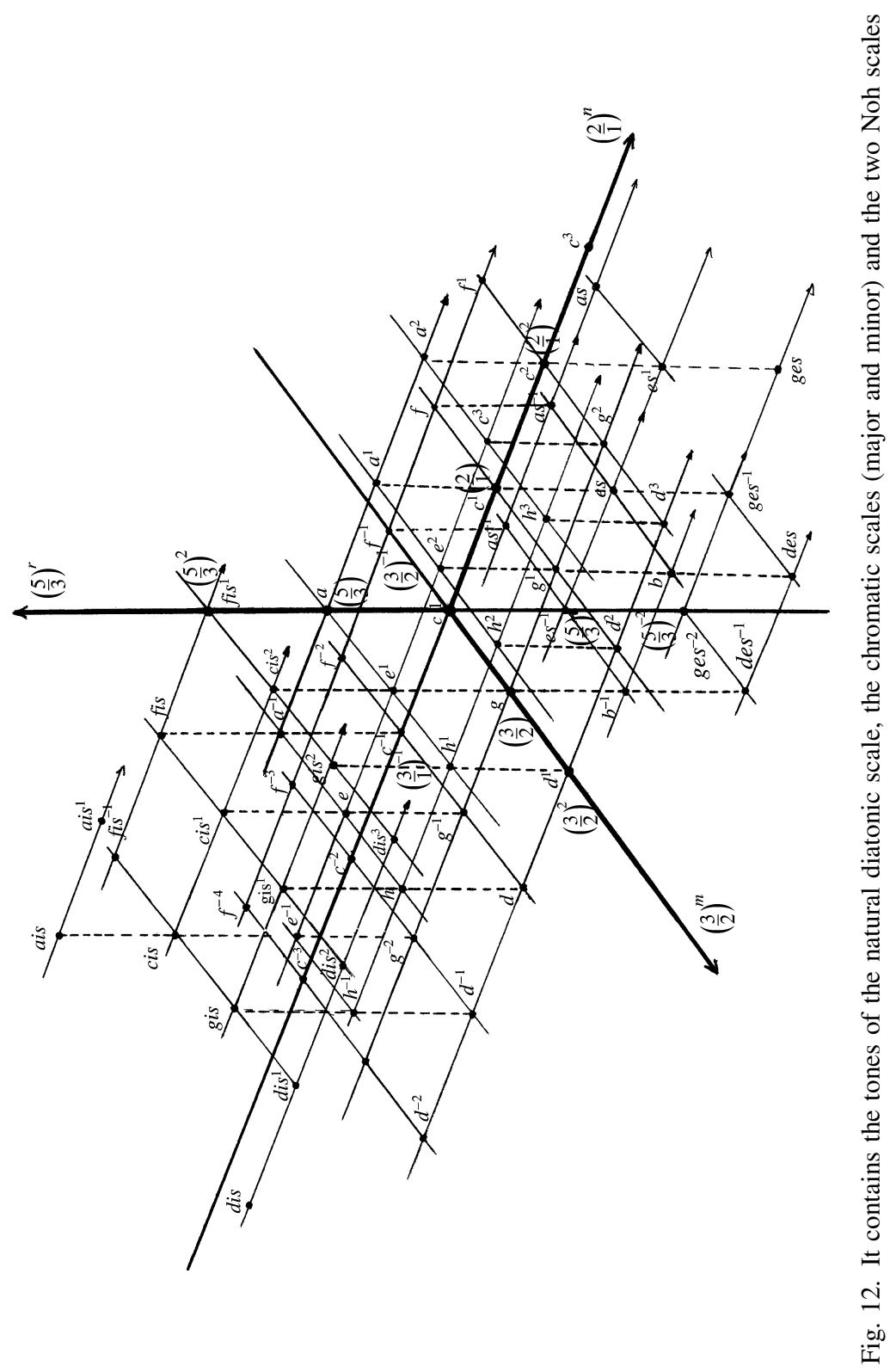




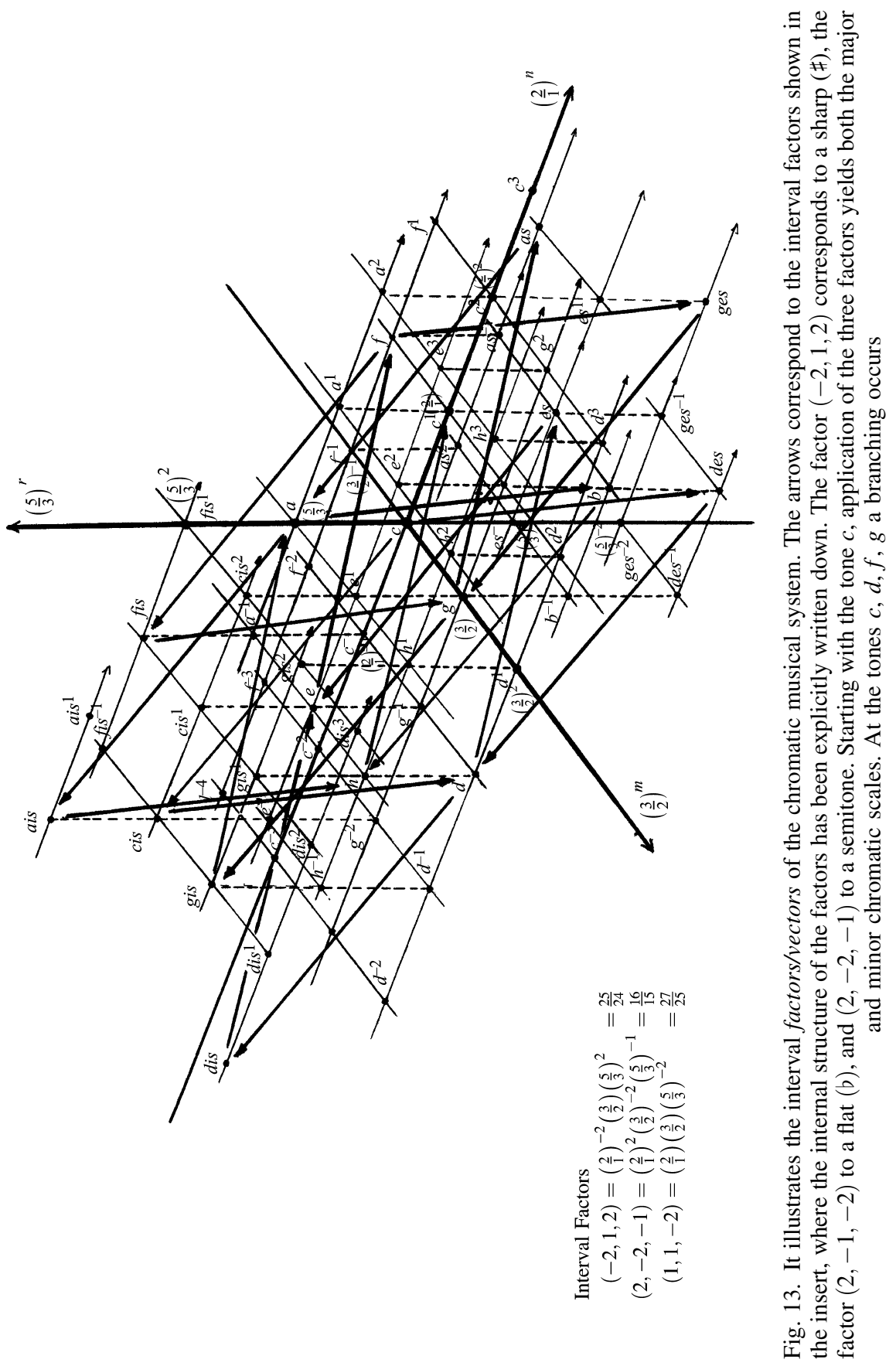




\section{Harmony-Triads (Dreiklang)}

The triads are given by

$$
\begin{gathered}
\{(n, m, r),(n-1, m+1, r+1),(n, m+1, r)\},(n, m, r) \equiv\left(\frac{2}{1}\right)^{n}\left(\frac{3}{2}\right)^{m}\left(\frac{5}{3}\right)^{r} \\
n, m, r=0, \pm 1, \pm 2, \ldots
\end{gathered}
$$

\section{Examples:}

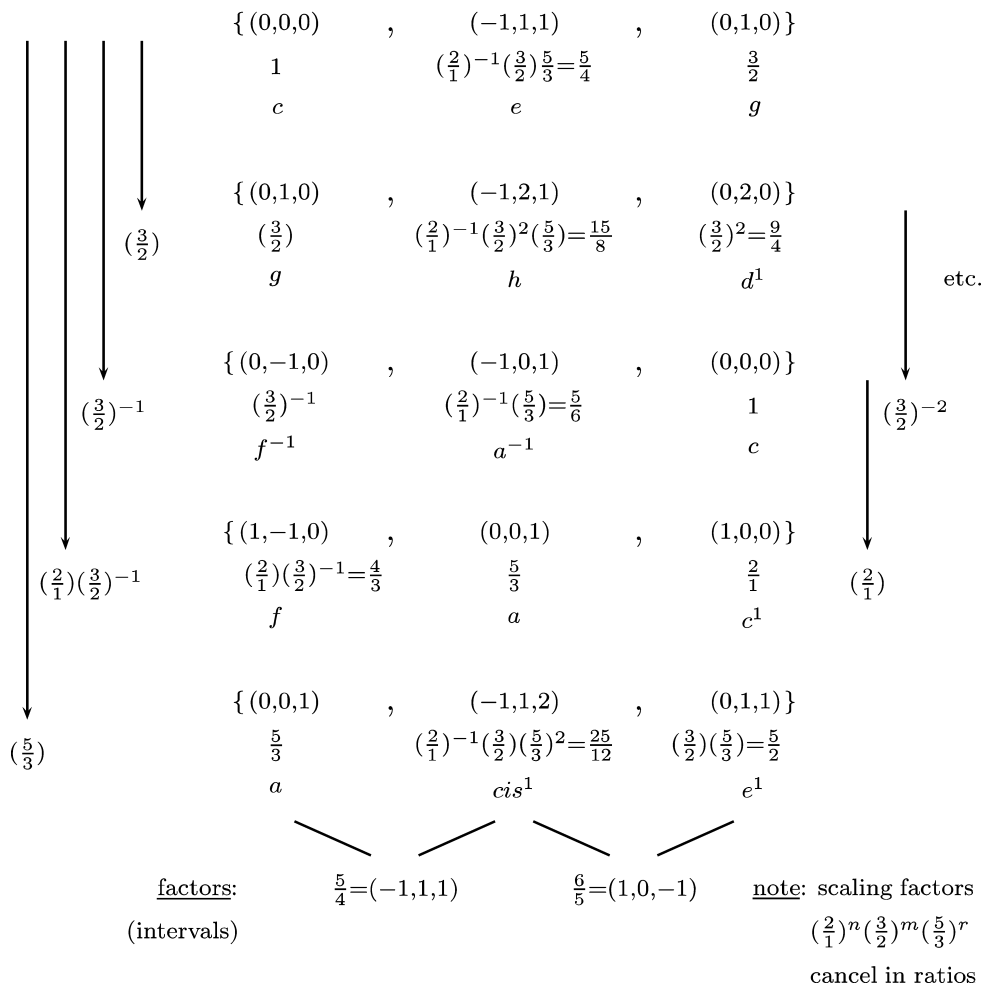

Fig. 14. It shows how triads can be easily identified graphically in the lattice given in Fig. 12 or Fig. 15 


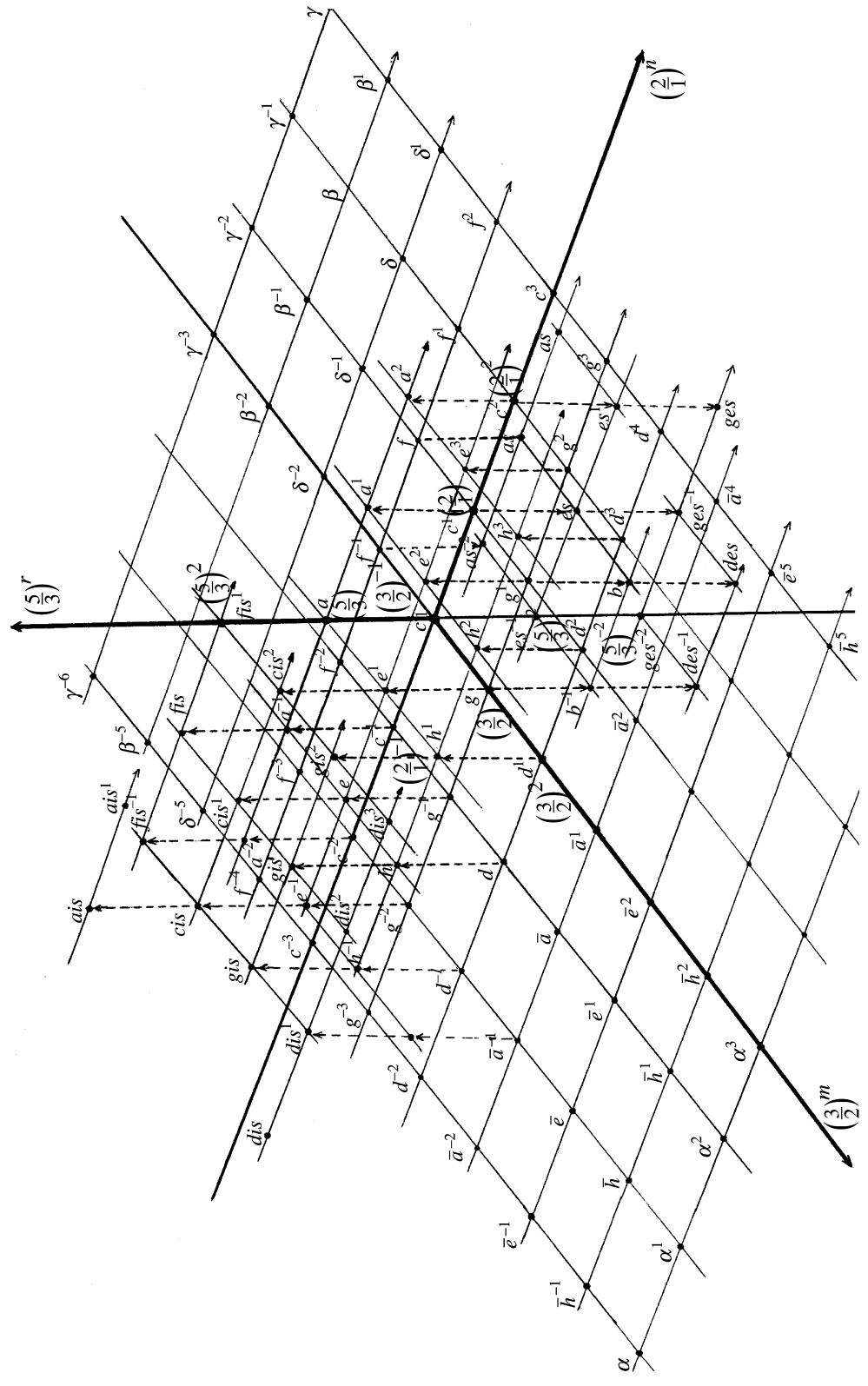

ํํㄹ

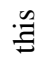

च

色

象

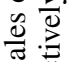

w

ङ

न है

है

Оิ10

过

3

ए

응

을

ह

ए

닝

옹

몸

$\circ$ 응

过

:

¿

吾

s.

氖艺

总

శี

a

छ

Ð

4

艺

플

क Z

as

v

in 


\section{References}

[1] Bergmann, L., Schaefer, C. (1954) Lehrbuch der Experimentalphysik, 1: Akustik. Walter de Gruyter, Berlin

[2] SHIgEO, K. (1984) The Traditional Music of Japan. Ongaku no Tomo-Sha, Tokyo

[3] Pierce, J. R. (1983) The Science of Musical Sound, p. 65. (Scientific American Library). Freeman, New York-San Francisco

[4] Riemann, H. (1970) Dictionary of Music, pp. 796-801. Angerer \& Co, London, Da Capo Press, New York (Music Theory Reprint Series)

Author's address: Prof. Bruno J. Gruber, Visiting Scientist, Institut für Meteorologie und Geophysik, Universität Wien, Hohe Warte 38, 1190 Wien, Austria. Permanent address: Emeritus, College of Science, Southern Illinois University, Mailcode 4403, Carbondale, IL 62901, USA. E-Mail: Gruber@siu.edu. 